\title{
The frequency of planets in multiple systems ${ }^{\star}$
}

\author{
M. Bonavita ${ }^{1,2}$ and S. Desidera ${ }^{1}$ \\ 1 INAF - Osservatorio Astronomico di Padova, Vicolo dell'Osservatorio 5, 35122 Padova, Italy \\ e-mail: silvano.desidera@oapd.inaf.it \\ 2 Dipartimento di Astronomia, Università di Padova, Italy
}

Received 31 October 2006 / Accepted 5 March 2007

\begin{abstract}
Context. The frequency of planets in binaries is an important issue in the field of extrasolar planet studies, because of its relevance in estimating of the global planet population of our Galaxy and the clues it can give to our understanding of planet formation and evolution. However, only preliminary estimates are available in the literature.

Aims. We analyze and compare the frequency of planets in multiple systems to the frequency of planets orbiting single stars. We also try to highlight possible connections between the frequency of planets and the orbital parameters of the binaries (such as the periastron and mass ratio.)

Methods. A literature search was performed for binaries and multiple systems among the stars of the sample with uniform planet detectability defined by Fischer \& Valenti (2005, ApJ, 622, 1102), and 202 of the 850 stars of the sample turned out to be binaries, allowing a statistical comparison of the frequency of planets in binaries and single stars and a study of the run of the planet frequency as a function of the binary separation.

Results. We found that the global frequency of planets in the binaries of the sample is not statistically different from that of planets in single stars. Even conservatively taking the probable incompleteness of binary detection in our sample into account, we estimate that the frequency of planets in binaries can be no more than a factor of three lower than that of planets in single stars. There is no significant dependence of planet frequency on the binary separation, except for a lower value of frequency for close binaries. However, this is probably not as low as required to explain the presence of planets in close binaries only as the result of modifications of the binary orbit after the planet formation.
\end{abstract}

Key words. stars: planetary systems - stars: binaries: general

\section{Introduction}

The increasing number of extra-solar planets discovered in binary or multiple stellar systems (Eggenberger et al. 2004; Raghavan et al. 2006; Desidera \& Barbieri 2007) suggests that planets can form and survive in a variety of stellar environments. Determining the frequency of planets in binaries is an important issue in the field of extrasolar planets studies, because of its relevance in estimating the global planet population of our Galaxy (more than half of the solar type stars are in binary or multiple systems as reported in Duquennoy \& Mayor 1991) and because of the clues it can give to our understanding of planet formation and evolution. The study of the properties of planets in binaries, as well as any difference to those of the planets orbiting single stars, could shed light on the effects from the presence of the companions.

A recent study by Desidera \& Barbieri (2007) shows that the mass distribution of short period planets in relatively tight binaries (separation $\leq 150-200 \mathrm{AU}$ ) is significantly different from that of planets orbiting the components of wide binaries and single stars. There are also other possible peculiar features of planets in tight binaries compared to planets orbiting single stars, such as a lack of long-period planets and multiple planets, that need confirmation. The properties of exoplanets orbiting the components of wide binaries are instead compatible with

$\star$ Table 8 and Appendix A are only available in electronic form at http://www . aanda. org those of planets orbiting single stars, except for a possible greater abundance of high-eccentricity planets.

This result implies that the formation and/or migration and/or dynamical evolution processes acting in the presence of a sufficiently close external perturber are modified with respect to single stars. Several scenarios can be devised to explain it, such as a different formation mechanism for planets in tight binaries (e.g. disk instability induced by dynamical perturbations, as proposed by Boss 2006), and enhanced migration and accumulation rate in the presence of a stellar companions (Kley 2000), and dynamical interactions after planet formation (Pfahl \& Muterspaugh 2006).

However, to better understand the cause of these anomalies and the origin itself of the planets in very close binaries (a challenge for current planet formation models) a key piece of information is missing: the frequency of planets in binaries as a function of the binary separation and compared to that of planets orbiting single stars. Determining the planet frequency in binaries is made difficult by the biases against binaries in most of the ongoing planet search surveys and by the incompleteness of binary and planet detections in these samples.

A first step in this direction has been made by Eggenberger et al. (2006), performing an adaptive-optics search for companions around stars with and without planets and without previously known stellar companions from the Coralie survey. This guarantees a fairly homogeneous binary detectability in their sample. However, the small number of objects (and the lack of confirmation of the physical association in a few cases at the 
time of the presentation of their preliminary results) did not allow them to make clear inferences on the planet frequency and in particular on possible differences as a function of the binary separations.

A much wider sample (850 stars vs the 110 stars studied by Eggenberger et al. 2006) that might be used for a study of planet frequency is the "Uniform Detectability" (hereafter UD) sample collected by Fischer \& Valenti (2005) (hereafter FV05). This sample is complete for detecting planets with radial velocity (hereafter RV) semi-amplitude $>30 \mathrm{~m} / \mathrm{s}$ and period $<4 \mathrm{yr}$. However, the binarity of stars in the UD sample has not been considered up to now.

Despite some incompleteness and biases concerning binarity, this sample can be considered valid to draw an independent measurement of the frequency of planets in binary stars, thanks to the completeness of planet detection and the large sample size. Thus, the binarity of the stars with uniform detectability was investigated in this work by searching some stellar catalogs listing stellar companions (Sect. 3). The result is a sub-sample of UD binaries, separated according to their different values of periastron and critical semimajor axis for dynamical stability of planetary orbits (see Holman \& Wiegert 1999) (Sect. 3.2). In this way it has been possible to compare the values of the frequency of planets in the two sub-samples (single stars and binary stars) and to verify a possible dependence of the frequency on critical semimajor axis and periastron (Sect. 4). The biases against binaries in the original selection sample, the completeness of the binary detection for stars with and without planets and their impact on the results are discussed in Sect. 4.1. The results are discussed further in Sect. 5, and in Sect. 6 we summarize our conclusions and offer future perspectives.

\section{The uniform detectability sample}

The uniform Detectability sample has been built by considering that, despite the detectability of the planets' changes from star to the next and from a survey to the other because of the different time span of the observations and different levels of RV errors, we can consider it complete for companions with velocity amplitudes $K>30 \mathrm{~m} / \mathrm{s}$ and orbital periods shorter than 4 years. Then, beginning from the initial target list, which included 1330 stars observed by the Lick, Keck, and Anglo Australian Surveys, FV05 selected a subsample of 850 stars that satisfy these entries provided that at least 10 observations spanning four years were available. Stars that were added after a planet was discovered by other groups were not included in the sample. However, stars independently present in one of these surveys were considered even if a planet was detected first by another group. Only planets with $K>30 \mathrm{~m} / \mathrm{s}$ and orbital periods shorter than 4-years were considered for the study of planet frequency. This corresponds to Saturn-mass planets for the shortest periods and Jupiter-mass planets for 4 year orbits.

\subsection{Changes in the UD sample}

During the analysis made for our work, we made some changes to the original UD sample, such as:

- we excluded 51 Peg because it was added to the considered target lists after the planet detection by Mayor \& Queloz (1995) (Marcy et al. 1997; Fischer 2005, private communications)

- $\tau$ Boo and $v$ And were marked in FV05 as "without planets", but the known companions fulfill all the selection criteria for the UD sample (Fischer 2005, private communication), so we included these stars as "with planets";

- HD 20782 hosts a planet detected after the compilation of the UD sample (Jones et al. 2006), but it is coherent with the UD requirements, so that this star has been considered as "with planets". The lack of this planet in the original UD sample confirms the hypothesis that high eccentricity $(e=0.93$ in this case) acts to make detection more difficult, as suggested by Cumming (2004);

- HD 18445 is flagged as "with planets" in the UD sample, probably because of a typo. It is not listed in the tables of stars with planets. The RV companion $\left(M \sin i=44 M_{J}\right)$ has a mass outside the giant planet range, and it was shown to be a $0.18 M_{\odot}$ star orbiting nearly pole-on by both astrometry (Halbwachs et al. 2000) and direct imaging (Beuzit et al. 2004), so we considered it as "without planets" (see Appendix A for details);

- we considered HD 196885 as "without planets" because the companion reported in FV05 was not confirmed by Butler et al. (2006) (see Appendix A for details);

The modified UD sample that is the result of those changes was then searched for companions, in order to build a sample of UD binaries.

\section{Searching for UD binaries}

In order to identify known or claimed companions for the stars included in the UD sample, we checked available sources listing stellar companions. Some of the most important sources are listed below:

- The Hipparcos and Thyco Catalogues (Perryman \& ESA 1997);

- The Catalogue of the Components of Double and Multiple Stars (CCDM) (Dommanget \& Nys 2002);

- The Washington Visual Double Star Catalog (WDS) (Worley \& Douglass 1997);

- Sixth Catalog of Orbits of Visual Binary Stars (Hartkopf \& Mason 2006);

- The Catalogue of Nearby Stars, Preliminary 3rd Version (C3) (Gliese \& Jahreiß 1991);

- Gould \& Chanamé (2004): New HIPPARCOS-based parallaxes for 424 faint stars;

- Nidever et al. (2002): Radial Velocities for 889 late-type stars;

- Allen et al. (2000): Wide binaries among high-velocity and metal-poor stars;

- Makarov \& Kaplan (2005): Proper motion derivatives of binaries;

- Tokovinin (1997): MSC - a catalogue of physical multiple stars $^{1}$

- Latham et al. (2002) Orbits of 171 single-lined spectroscopic binaries;

- Halbwachs (1986) Common proper motions stars in AGK3;

- The revised NLTT catalogue (Salim \& Gould 2003);

- Valenti \& Fischer (2005) Spectroscopic properties of cool stars. I. (VF05);

- The Tycho Double Star Catalogue (TDSC) (Fabricius et al. 2002);

1 Updated version available at www. ctio.noao. edu/ atokovin/stars/ 
- A Catalog of Northern Stars with Annual Proper Motions Larger than 0.15" (LSPM-NORTH Catalog)" (Lépine \& Bongiorno 2007)

- SB9: The ninth catalogue of spectroscopic binary orbits (Pourbaix et al. 2004)

- 2MASS All Sky Catalog of point sources (Cutri et al. 2003) has been used only for deriving $J H K$ photometry (used for mass determination) and for common proper motion confirmation, not for a search for further companions.

We also consider additional references for individual objects (see Table 8 and Appendix A).

\subsection{Selection criteria}

After this search, we excluded from our UD binary sample the stars with non confirmed companions and with companions listed in CCDM but with inconsistent proper motions (and without other indication of binarity found in literature), and considered those stars as singles. Stars with long-term RV and/or astrometric trends were included in the binary sample, on the basis of the dynamical evidence of a companion. The RV trends we included (from Nidever et al. 2002) cause an overall RMS of RV faster than $100 \mathrm{~m} / \mathrm{s}$ that cannot be due to planetary companions. We also included stars with brown dwarf companions in the sample of binaries. At small separation, the existence of the brown dwarf desert (see Butler et al. 2006) guarantees little ambiguity in the classification of an object as a massive planet or a brown dwarf (a couple of individual cases are listed in Appendix A). At large separations, where brown dwarfs companions are probably more frequent (Gizis et al. 2001), this ambiguity should be taken into account but is again limited to a few individual cases, e.g. HD 206860, which has a T dwarf companion of mass $0.021 \pm 0.09 M_{\odot}$ according to Luhman et al. (2007), smaller than the mass limit for planetary companions adopted by Butler et al. (2006). The small number of brown dwarf companions makes this issue irrelevant for the global results.

\subsection{The sub-sample of UD binaries}

The properties of the UD binaries, selected from the modified UD sample, are listed in Table 8 . The stars with both components included in the UD sample are listed twice, otherwise only the star under planet scrutiny is listed. If more than one companion is known, we report only the closer one, because of its stronger influence on planetary formation/stability. For hierarchical triple (or higher-order multiplicity) systems, for which the isolated star is included in the UD sample, we sum the mass of the closest pair to consider its effective dynamical influence. The minimum mass is listed for single-lined spectroscopic binaries.

For each object we report:

- the HD number;

- the projected separation;

- the eccentricity, if the binary orbit is available. Otherwise we assume $e=0.31$, which is a median value between those adopted by Fischer \& Marcy (1992) and Duquennoy \& Mayor (1991);

- the semi major axis in AU. For those stars for which the binary orbit is not available, the semimajor axis was estimated from the projected separation $a(\mathrm{AU})=1.31 * \rho(\operatorname{arcsec}) * d$ (pc) (Fischer \& Marcy 1992; Duquennoy \& Mayor 1991);

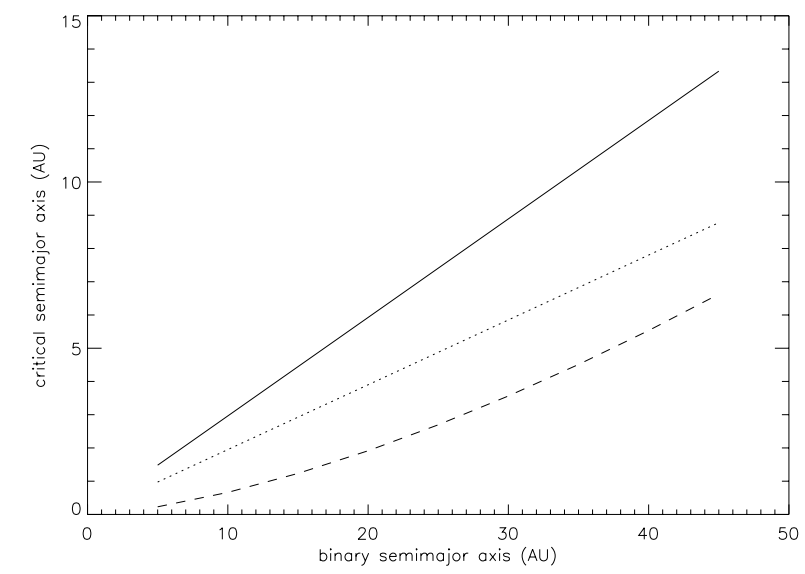

Fig. 1. Values of $a_{\text {crit }}$ (solid line) and $a_{\text {cross }}$ (dashed line) and $a_{\text {tid }}$ (dotted line), versus binary semimajor axis, for $e=0.31, M_{\mathrm{com}}=0.5 M_{\odot}$, and $M_{\text {obj }}=1 M_{\odot}$.

- the mass of the objects, from Fischer \& Valenti (2005) (hereafter $\mathrm{VF} 05)^{2}$;

- the companion mass

a) from VF05 if both components are included in the UD sample;

b) calculated with the mass-luminosity relations derived by Reid \& Gizis (1997); Delfosse et al. (2000);

c) from other literature sources (listed in the Table caption); - the critical semi-major axis for dynamical stability of planetary companions on circular orbits coplanar with the binary orbit, calculated using the equation

$$
\begin{array}{r}
a_{\text {crit }}=\left(0.464-0.380 \mu-0.631 e_{\text {bin }}+0.586 \mu e_{\text {bin }}\right) a_{\text {bin }} \\
+\left(0.150 e_{\text {bin }}^{2}-0.198 \mu e_{\text {bin }}^{2}\right) a_{\text {bin }}
\end{array}
$$

by Holman \& Wiegert (1999). In Eq. (1): $\mu=\frac{M_{\mathrm{com}}}{M_{\mathrm{obj}}+M_{\mathrm{com}}}, a_{\text {bin }}$ is the semi major axis and $e_{\text {bin }}$ is the eccentricity of the binary orbit.

We chose $a_{\text {crit }}$ as a reference value, because it is a physical quantity that represents, better than the semi-major axis or the projected separation, the dynamical effects of the presence of the companion first on the circumstellar region and then on planet formation and stability. This feature, in fact, includes both the orbital parameters and the mass ratio, and represents the maximum value of the semimajor axis for stable planetary orbits around the planet hosts. The value of $a_{\text {crit }}$ is higher than the limit of the region in which the encounter velocities of planetesimal is small enough to allow the accretion of kilometer-sized planetesimals ( $a_{\text {cross }}$, Thébault et al. 2006). The radius of tidal truncation of the circumstellar disk $a_{\text {tid }}$ (Pichardo et al. 2005; Pfahl \& Muterspaugh 2006) is intermediate between $a_{\text {crit }}$ and $a_{\text {cross. }}$. Figure 1 shows the values of $a_{\text {crit }}$ (solid line), $a_{\text {cross }}$, and $a_{\text {tid }}$, versus binary semimajor axis, for fixed values of eccentricity and masses $\left(e=0.3, M_{\mathrm{com}}=0.5 M_{\odot}\right.$, and $\left.M_{\mathrm{obj}}=1 M_{\odot}\right)$.

\section{Results and selection effects}

The result of this search for companions of the UD stars is a subsample of 202 objects, 15 of those having planets, so the global frequency of planets in the UD binary sample is $7.4 \%$. The frequency of planets in the UD single stars sub-sample is $5.3 \%$ (see

\footnotetext{
${ }^{2}$ The exceptions are listed in Appendix A.
} 
Table 1). The two frequencies are compatible within their errors $^{3}$, and the slightly higher value of the global frequency in the binary sub-sample is probably due to some incompleteness in the sample, which is discussed in Sect. 4.1.

The rather large sample size allows us to divide stars in some sub-samples according to different values of critical semimajor axis for dynamical stability of planets (hereafter $a_{\text {crit }}$; see Eq. (1)). All the stars with RV and/or astrometric trend and without direct imaging identification or full orbit characterization (37 objects) were included in the closest bin, as it is likely that the companion responsible for the trend has a small separation.

We also binned the UD binary sample according with the periastron because this allowed us to make a direct comparison with theoretical expectations, such as those of Pfahl \& Muterspaugh (2006). The resulting values of the frequency are listed in Tables 1 and 2, together with the characteristics of each sample. In Table 1 we also show the values of frequency for the complete UD binary sample and for the UD single sub-sample. Figures 2-3 show $a_{\text {crit }}$ and periastron vs mass ratios for the binaries of the sample with and without planets.

These figures suggest that the binary components hosting planets usually have low-mass secondaries, for both low and high separation values. This item is confirmed by Fig. 4 which shows the histogram of the mass-ratio values for the two populations. In order to better investigate the dependence of the planet frequency on the binary mass-ratio, we performed a Kolmogorov Smirnov Test (hereafter KST) on the two populations. The resulting value for the KST probability is $\sim 11 \%$ for the entire sample (excluding objects with trends, for which the value of the secondary mass is unknown) and $\sim 10 \%$ for the stars with $a_{\text {crit }}>20 \mathrm{AU}$. The difference between the distributions is not significant enough to allow us to confirm or reject the hypothesis that the presence of planets is favored in binaries with a low value of the mass ratio. This feature, as discussed in Sect. 4.1.2, could also be due to how the stars hosting planets are preferentially searched for low-mass companions, and this is probably one of the causes of their lower observed values of the mass ratio. On the other hand, the lack of planet detections up to now in the ongoing RV planet search using SARG at TNG (Desidera et al. 2006), which targets only binaries with similar components and with a typical separation between 100 to $400 \mathrm{AU}$, suggests that the mass ratio might be an important parameter for the occurrence of planets. Therefore, the possible role of the mass ratio on the frequency of planets remains an open point that requires further investigations.

\subsection{Completeness and selection effects}

These results suggest that the planet frequency in binaries and single stars is similar. However, selection effects concerning binaries in the original definitions of the Lick, Keck, and AAT samples and possible incompleteness of binary detection might alter the results; we discuss them here in order to better estimate the frequency of planets in binaries.

3 The errors reported in Tables 1 and 2 are calculated with the equation

$\sigma_{f}=\left(N_{\text {planets }}^{-1 / 2}+N_{\text {star }}^{-1 / 2}\right) *\left(\frac{N_{\text {planets }}}{N_{\text {stars }}}\right)$.

They do not include the additional error due to the incompleteness of binary detection.

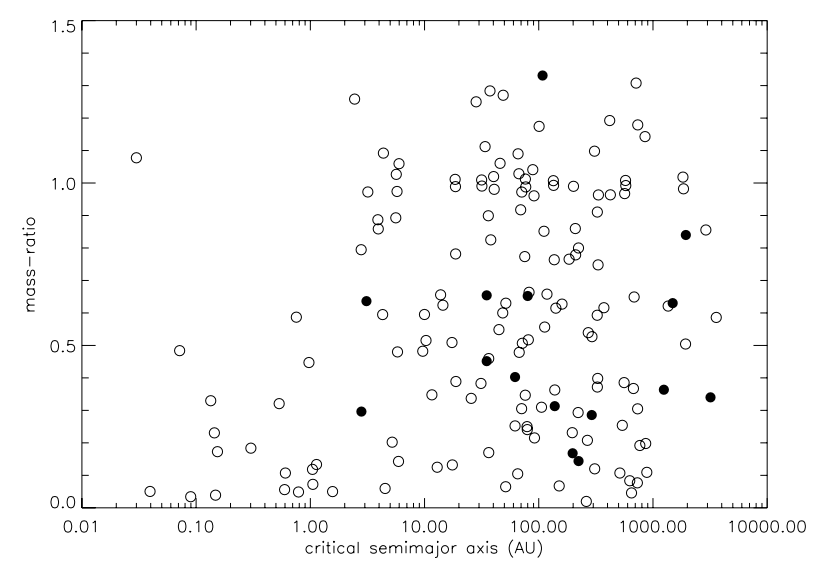

Fig. 2. Critical semimajor axis (from Holman \& Wiegert 1999) vs. mass-ratio for the binaries with planets (filled circles) and without planets (open circles) in the UD sample.

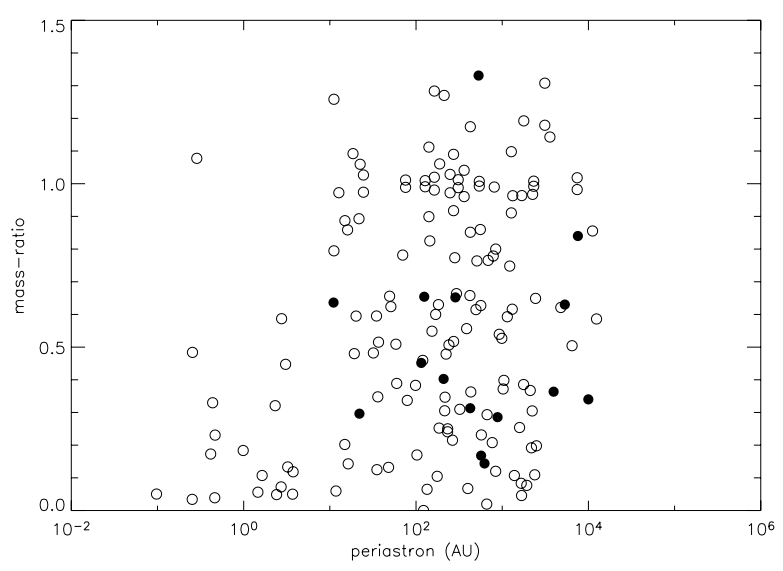

Fig. 3. Periastron vs. mass-ratio for the binaries with planets (filled circles) and without planets (open circles) in the UD sample.

Table 1. Frequency of planets in binaries with different values of $a_{\text {crit }}$.

\begin{tabular}{cccc}
\hline \hline$a_{\text {crit }}$ & $N_{\text {star }}$ & $N_{\text {planets }}$ & $\frac{N_{\text {planets }}}{N_{\text {stars }}}$ \\
\hline$<20 \mathrm{AU}$ & 89 & 2 & $0.022 \pm 0.018$ \\
$20-50 \mathrm{AU}$ & 18 & 2 & $0.111 \pm 0.105$ \\
$50-100 \mathrm{AU}$ & 24 & 2 & $0.083 \pm 0.076$ \\
$100-250 \mathrm{AU}$ & 26 & 4 & $0.154 \pm 0.107$ \\
$>250 \mathrm{AU}$ & 45 & 5 & $0.111 \pm 0.066$ \\
\hline UD Singles sub-sample & 647 & 34 & $0.053 \pm 0.011$ \\
Entire UD binary sub-sample & 202 & 15 & $0.074 \pm 0.024$ \\
\hline
\end{tabular}

\subsubsection{Estimate of the missing binaries}

The first issue to consider is the completeness of the binary census in the sample. We can derive an upper limit to the incompleteness by assuming that the stars in the UD sample have the same binary frequency as in Duquennoy \& Mayor (1991) (57\%). The number of missing binaries in the sample would then be $\sim 296^{4}$.

To derive a lower limit to the frequency of planets in binaries, we assume that all the "missing" binaries are without planets.

\footnotetext{
${ }^{4}$ For the estimate of the number of unrecognized binaries, we excluded (and then consider them as single stars) the stars with brown dwarfs companions, because the substellar companions are not included in the statistical analysis by Duquennoy \& Mayor (1991); hence, the number of binaries found in the UD sample decreases to 188 .
} 


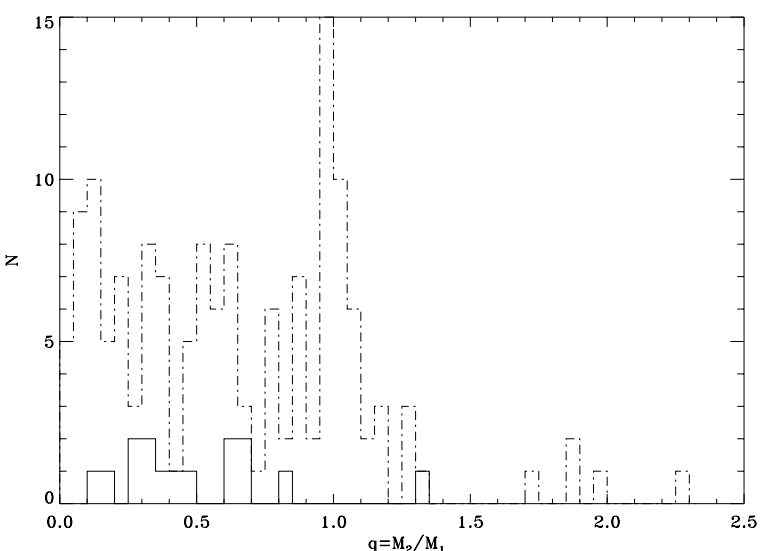

(a)

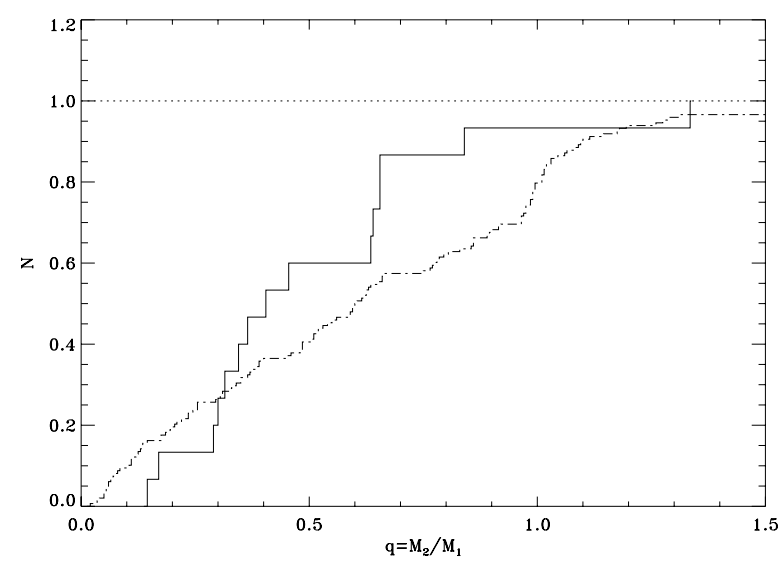

(b)

Fig. 4. Distribution of mass-ratio values for star with (solid line) and without (dashed line) planets. Panel a) shows the histogram and panel b) the cumulative distribution.

Table 2. Frequency of planets in binaries with different values of periastron.

\begin{tabular}{cccc}
\hline \hline Periastron & $N_{\text {star }}$ & $N_{\text {planets }}$ & $\frac{N_{\text {planets }}}{N_{\text {stars }}}$ \\
\hline$<50 \mathrm{AU}$ & 81 & 2 & $0.025 \pm 0.020$ \\
$50-200 \mathrm{AU}$ & 28 & 2 & $0.071 \pm 0.064$ \\
$200-500 \mathrm{AU}$ & 30 & 3 & $0.100 \pm 0.076$ \\
$500-1000 \mathrm{AU}$ & 23 & 4 & $0.174 \pm 0.123$ \\
$>1000 \mathrm{AU}$ & 39 & 4 & $0.103 \pm 0.068$ \\
\hline
\end{tabular}

We then obtain $f_{\text {bin }}=3.09 \pm 0.94 \%$ and $f_{\text {sin }}=9.31 \pm 0.69 \%$; i.e. the frequency of planets in binaries cannot be less than one third of that of planets orbiting single stars. We stress that this is a very conservative lower limit on the frequency of planets in binaries (an upper limit on the incompleteness of the binary detection in the sample), because the UD sample has selection biases against binarity. In fact the input target lists exclude spectroscopic binaries and binaries with separations less than 2 arcsec known at the time of the target selection (see Jones et al. 2002; Marcy et al. 2005; Wright et al. 2004). Therefore we expect that the absolute binary frequency in the UD sample is significantly lower than the unbiased one derived by Duquennoy \& Mayor (1991); the distribution of the orbital parameters and mass ratio of the binaries in the UD sample should also be different with respect to unbiased samples.
To take this selection bias in account, we did a Monte Carlo simulation making an estimate of the fraction of binaries with $\rho<2^{\prime \prime}$ expected on the basis of the period and mass-ratio distribution assumed by Duquennoy \& Mayor $(1991)^{5}$. In this way we found that $\sim 45.7 \%$ of the binaries predicted by Duquennoy $\&$ Mayor (1991) have $\rho<2$ " for the distance distribution of the stars of the UD sample, so we expect that a significant fraction of the "missing" binaries are given by stars excluded at the time of the target selection because of their small separation.

Combining these features, we find that the total number of binaries expected in the UD sample with a separation $>2$ arcsec is 221 . Our census yields 138 binaries (120 without considering wide companions orbiting close pairs with a separation $<2$ arcsec) so there should be 83 missing binaries (95 without considering wide companions in triple systems) with separations $>2$ arcsec.

These numbers are expected to hold if there are no biases besides the exclusion of binaries with separations smaller than 2 arcsec in the UD sample. However, it is possible that the stars that were added later to the samples for specific reasons (e.g. high metallicity, Butler et al. 2000; Tinney et al. 2003) have different selection criteria concerning binarity. In some individual cases, the inclusion in the input target lists of certain types of systems is favored: a few binary systems with similar components were probably included in the sample after dedicated studies of chemical abundances differences between the components (Gratton et al. 2001; Martín et al. 2002). Unfortunately, we do not have enough information about the details of the building of the sample. We do expect that this only plays a minor role in the global binary statistics.

\subsubsection{Completeness of the binarity of planet hosts vs. non planet hosts}

The role of completeness in binary detection would be minor for our purposes if the completeness itself were independent of the occurrence of planets. Unfortunately, this is not the case. Planet hosts are systematically searched for companions after planet discoveries. Therefore, the completeness of the binarity of planet hosts is certainly greater than that of stars without planets. This bias could cause a spurious increase in planet frequency in binaries and should be carefully addressed. We proceeded as follows. We considered the binarity of planet hosts in the UD sample (including those with $K<30 \mathrm{~m} / \mathrm{s}$ and/or $P>4 \mathrm{yr} ; 21$ objects overall). If the binarity was found on the basis of dedicated studies after planet discoveries, the star was classified as single; if instead the binarity was known independently of the planet discovery (e.g. inclusion in WDS, CCDM, Hipparcos, detection of astrometric and RV trends, etc.), the star was kept as a binary. This results in a change in binary status for 7 stars ( 4 with

5 The distribution in orbital period assumed by Duquennoy \& Mayor (1991) is

$f(\log P)=C \exp \frac{-(\log P-\overline{\log P})^{2}}{2 \sigma_{\log P}^{2}}$

where $\overline{\log P}=4.8, \sigma_{\log P}=2.3$ and $P$ is in days. For the mass-ratio $q=M_{2} / M_{1}$ they assume

$\xi(q)=k \exp \left\{\frac{-(q-\mu)^{2}}{2 \sigma_{q}^{2}}\right\}$

where $\mu=2.3, \sigma_{q}=0.42$ and $k=18$ for their sample of G-Dwarfs. 
Table 3. Frequency of planets in binaries with different values of $a_{\text {crit }}$, without considering as a binary the planet-host whose companions were discovered thanks to dedicated follow up after planet detection.

\begin{tabular}{cccc}
\hline \hline$a_{\text {crit }}$ & $N_{\text {star }}$ & $N_{\text {planets }}$ & $\frac{N_{\text {planets }}}{N_{\text {stars }}}$ \\
\hline$<20 \mathrm{AU}$ & 89 & 2 & $0.022 \pm 0.018$ \\
$20-50 \mathrm{AU}$ & 18 & 2 & $0.111 \pm 0.105$ \\
$50-100 \mathrm{AU}$ & 22 & 2 & $0.091 \pm 0.083$ \\
$100-250 \mathrm{AU}$ & 23 & 2 & $0.087 \pm 0.079$ \\
$>250 \mathrm{AU}$ & 43 & 3 & $0.070 \pm 0.051$ \\
\hline UD Singles sub-sample & 654 & 38 & $0.058 \pm 0.011$ \\
Entire UD binary sub-sample & 195 & 11 & $0.056 \pm 0.021$ \\
\hline
\end{tabular}

UD planets and 3 with non-UD planets). In this way we obtained a binary sample that is less complete than the original one but without biases favoring the binarity of planet hosts. The revised frequency of planets in binaries then becomes $11 / 195=0.056$ and the corresponding for single stars 38/654 $=0.058$ (the results for all the $a_{\text {crit }}$ bins are reported in Table 3). The difference with respect to the full sample indicates that indeed the dedicated searches for companions around planet hosts somewhat alters the results but the frequencies of planets in binaries and in single stars remain similar.

\subsubsection{Dependence on separation}

The completeness of binarity in this sample is probably a function of the separation. At small separations, the inclusion of stars with RV and an astrometric trend probably allows a fairly high completeness level. In fact, most of the binaries recently discovered by means of deep adaptive optics imaging (e.g. HD 13445; HD 161797, HD 190406, HD 196885; see App. A for references) would have been included as binaries in this study thanks to their dynamical signatures, even without the direct imaging identification. Pairs with a small magnitude difference in separation between 0.2 to 10 arcsec should have been detected by Hipparcos (Quist \& Lindegren 2000). Wide binaries $(\rho>5-10$ arcsec) are more easily discovered and then included in CCDM and WDS even for larger magnitude differences. Intermediate values of separation (e.g. 1 to 5 arcsec) are probably the most incomplete ones, as the detection of a lowmass companion requires dedicated high-resolution imaging that is not available for all the stars, and the companions are not expected to produce detectable RV or astrometric signatures.

\subsubsection{Effects of incomplete information on orbit and masses of the companions}

The determination of $a_{\text {crit }}$ requires the availability of the full binary orbit and an estimate of the mass the companion. All these quantities are unknown for the 37 stars with astrometric and/or RV trends . In any case, we included these objects in the binary statistic because of the significance high level of the trends as resulting from the original works by Makarov \& Kaplan (2005) and Nidever et al. (2002). Furthermore, several objects present both the astrometric and RV signatures, which further confirms their nature as multiple objects. From the timescales of the detected orbital motion, we can reasonably infer a period short enough to allow the inclusion of these stars in our closest bin $\left(a_{\text {crit }}<20 \mathrm{AU}\right)$. However, any more detail on the separation distribution of these companions cannot be determined, which representing a major limitation for studying of the details run of planet frequency vs. $a_{\text {crit }}$ at small separations.
Table 4. Frequency of planets in binaries with different values of $a_{\text {crit }}$ for the volume-limited sample.

\begin{tabular}{cccc}
\hline \hline$a_{\text {crit }}$ & $N_{\text {star }}$ & $N_{\text {planets }}$ & $\frac{N_{\text {planets }}}{N_{\text {stars }}}$ \\
\hline$<20 \mathrm{AU}$ & 21 & 2 & $0.095 \pm 0.088$ \\
$>20 \mathrm{AU}$ & 23 & 2 & $0.087 \pm 0.079$ \\
\hline VLUD Singles sub-sample & 85 & 8 & $0.094 \pm 0.043$ \\
Entire VLUD binary sub-sample & 44 & 4 & $0.091 \pm 0.059$ \\
\hline
\end{tabular}

Table 5. Frequency of planets in binaries with different values of periastron for the volume limited sample.

\begin{tabular}{cccc}
\hline \hline Periastron & $N_{\text {star }}$ & $N_{\text {planets }}$ & $\frac{N_{\text {planets }}}{N_{\text {stars }}}$ \\
\hline <50 AU & 19 & 2 & $0.105 \pm 0.098$ \\
>50 AU & 25 & 2 & $0.080 \pm 0.072$ \\
\hline
\end{tabular}

For the stars where only the projected separation is available, we have considered an approximate values for the semimajor axis and eccentricity values from the adopted statistical relations. Even if for individual objects this approximation could be quite different from the real value, we expect that statistically it would give a realistic representation of the true semimajor axis and periastron distributions.

\subsection{The volume-limited sample}

As a further investigation of the role of the biases mentioned in Sect. 4.1 in our results, we have selected a volume-limited sample (hereafter VLUD), including the stars within a distance of $18 \mathrm{pc}$ from the Sun, analogous with FV05. The selection effects considered in Sect. 4.1 are expected to be smaller for the VLUD sample. In fact, the census of companions around stars in this sample is expected to be more complete than that of the global sample because the closest stars were in general more carefully searched for stellar companions, and at close distance the 2 arcsec limit corresponds to a smaller physical separation (36 AU at $18 \mathrm{pc}$ ). The small distance also favors overlap between different detection techniques. Indeed, only 1 star with astrometric trend and without direct imaging identification is included in the VLUD sample.

The volume-limited UD sample includes 129 stars, of which 44 are known binaries. The fraction of stars with planets in the VLUD sub-sample is $9.1 \%$ for binaries and $9.4 \%$ for single stars. We selected some bins in $a_{\text {crit }}$ and periastron also for the VLUD sample but, because of the small number of stars included in this sample, we considered only 2 ranges of values. Tables 4 and 5 show the frequency values obtained for the VLUD sub-samples. Statistical error bars are larger for the VLUD sample because of the small number of objects but the frequency of planets in single stars and in binaries is again similar.

A possibly interesting difference with respect to the full UD sample is that the frequency of planets in tight binaries $\left(a_{\text {crit }}<20 \mathrm{AU}\right)$ is not lower than in wide binaries and single stars. This might be explained looking at Fig. 5. The panels show the distribution of $a_{\text {crit }}$ for the lowest bin in $a_{\text {crit }}$ for the complete UD sample (upper panel) and for the VLUD sample (lower panel). In both panels, the first column on the left contains the stars included as binaries only because of the long-term $\mathrm{RV}$ or astrometric trends (i.e. mass and separation of the companions not known). From these histograms we can easily see that the percentage of the stars without definite orbital characteristics (thus included only on the basis of dynamical signatures) is 


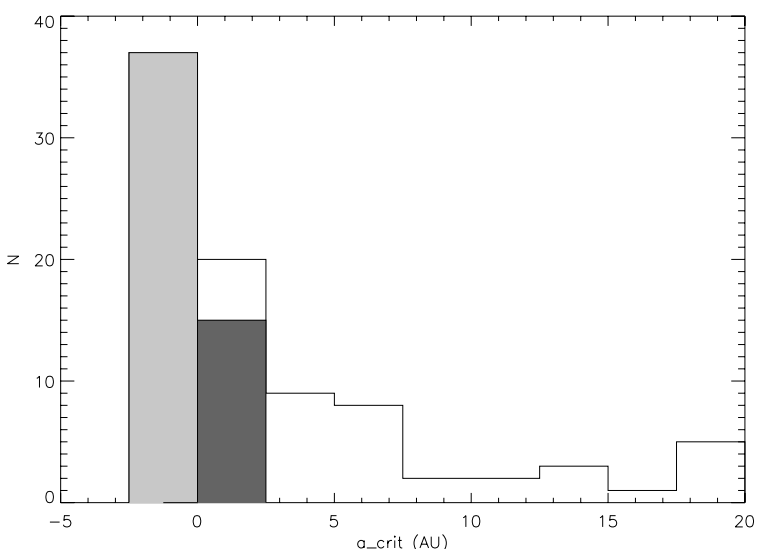

(a)

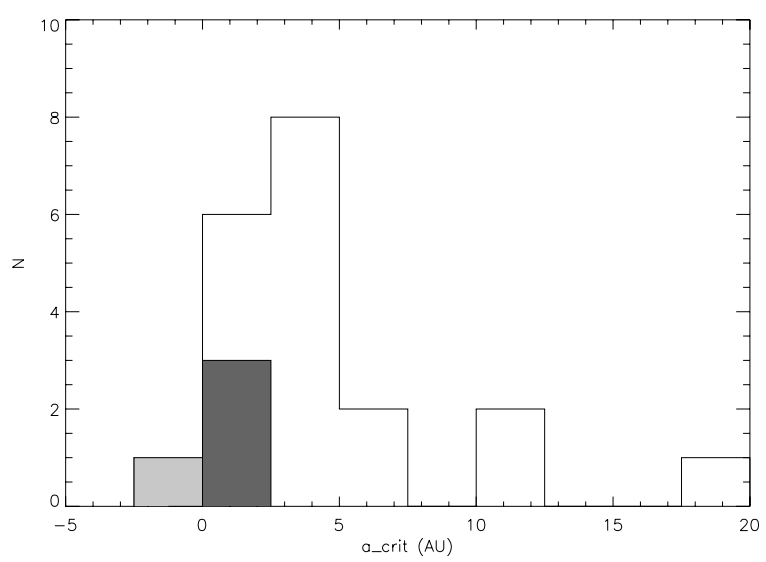

(b)

Fig. 5. Distribution of $a_{\text {crit }}<20 \mathrm{AU}$ for the complete UD binary sample a) and for the VLUD sample b). In both panels, the light grey column contains the stars with-long term RV or astrometric trends, arbitrarily placed at $a_{\text {crit }}<0$ for display purposes. The dark grey column represents the contribute of spectroscopic binaries.

much larger in the complete UD sample $(37 / 89, \sim 42 \%)$ with respect to the VLUD sample in which just one object, HD 120780 , is included only thanks to its astrometric trend. At the same time, for the systems for which $a_{\text {crit }}$ can be derived, the distribution of the $a_{\text {crit }}$ values for the VLUD sample is centered on the second bin $\left(2.5<a_{\text {crit }}<5.0 \mathrm{AU}\right)$, while in the complete sample there is a relatively large number of binaries (at least 20, excluding those with only RV or astrometric trends) for which $a_{\text {crit }}$ is smaller than $\sim 2.5 \mathrm{AU}$, the separation limit corresponding to $P=4 \mathrm{yr}$ for solar-type systems. Therefore a lower frequency for planets in these systems is expected, as only a part of the separation range considered here can host planets on stable orbits.

\section{Discussion}

\subsection{Global estimate of frequency of planets in binary stars}

Using a sample made of binaries with uniform planet detectability, we compared the frequency of planets in binaries to that of planets orbiting single stars. Looking at the whole binary sample we can conclude that the frequency of planets in binaries is not statistically different from planets orbiting single stars. With very conservative assumptions on the incompleteness of binary detection, we find that the frequency of planets in the binaries of the sample cannot be lower by more than a factor of three
Table 6. Probability of observing 2 binaries with planets in a sample of 89 (resp. 21) binaries in the UD (resp. VLUD) sample with $a_{\text {crit }}<$ $20 \mathrm{AU}$, for different planet frequencies.

\begin{tabular}{ccc}
\hline \hline Frequency & $\begin{array}{c}\text { Probability } \\
\text { full sample }\end{array}$ & $\begin{array}{c}\text { Probability } \\
\text { VLUD sample }\end{array}$ \\
\hline $0.1 \%$ & $0.3 \%$ & $0.02 \%$ \\
$1 \%$ & $16.3 \%$ & $1.7 \%$ \\
$5 \%$ & $11.3 \%$ & $19.8 \%$ \\
$10 \%$ & $0.4 \%$ & $28.4 \%$ \\
\hline
\end{tabular}

compared to planets orbiting single stars. When considering samples that are less affected by selection biases such as the VLUD sample, or excluding only the binaries detected thanks to dedicated follow-up after planet discoveries, that cause a spurious increase of the binary fraction of planet hosts, we found that the two frequencies are indeed very similar. This is qualitatively consistent with the preliminary results of Eggenberger et al. (2006).

An important point to consider is that this conclusion applies to the kind of binaries that are included in UD sample, i.e. with a separation distribution biased against close binaries, and cannot be directly applied to samples with unbiased binary distribution. Furthermore, it applies to the kind of planets fulfilling the requirements for inclusion in the UD sample, i.e. $P<4 \mathrm{yr}$ and $K>30 \mathrm{~m} / \mathrm{s}$. It is possible that e.g. the effects of binarity on planets in wider orbits are stronger.

\subsection{Dependence on the binary separation}

The size of our sample allowed us to divide it into some subgroups according to the value of the critical semimajor axis for the dynamical stability. In this way we can argue that there is no significant dependence of the frequency on $a_{\text {crit }}$ (and on the periastron) except for companions with $a_{\text {crit }}$ less than $20 \mathrm{AU}$ (that corresponds to a separation $<50-100 \mathrm{AU}$, depending on the mass ratio of the components). This result makes stronger the conclusion by Desidera \& Barbieri (2007), who reported that the presence of distant companions (separation $>300-500 \mathrm{AU}$ ) does not significantly affect the process of planet formation, as the mass and period distribution of planets in such wide binaries are similar to those of planets orbiting single stars (Desidera \& Barbieri 2007), and the frequency of planets is also similar (this work).

What happens at small separations is more crucial to understanding the role of companions in planet formation. Desidera $\&$ Barbieri (2007) found that the properties of planets in close binaries, in particular the mass distribution, are different from those orbiting single stars and components of wide binaries ${ }^{6}$. We showed in this study indication for a lower frequency of planets in tight binaries.

The frequency of planets in close binaries can be used to further investigate how these planets formed and the origin of their anomalous properties. Indeed, Pfahl \& Muterspaugh (2006) shows that knowing the value of the frequency of planets in close binaries ${ }^{7}$ should allow two alternative formation scenarios to be distinguished. A low frequency (about $0.1 \%$ but with an uncertainty of about one order of magnitude, so we can consider $1 \%$ as a limit-value) would be compatible with dynamical interactions that cause the formation of the tight binary after planet formation.

\footnotetext{
6 Note that the definition of tight binaries used by Desidera \& Barbieri (2007) $\left(a_{\text {crit }}<75 \mathrm{AU}\right)$ is different than the one adopted here.

7 Defined as those binaries with semi-major axis less than $50 \mathrm{AU}$.
} 
Table 7. Probability of observing 2 binaries with planets in a sample of 81 (resp. 19) binaries in the UD (resp. VLUD) sample with periastron $<50 \mathrm{AU}$, for different planet frequencies.

\begin{tabular}{ccc}
\hline \hline Frequency & $\begin{array}{c}\text { Probability } \\
\text { full sample }\end{array}$ & $\begin{array}{c}\text { Probability } \\
\text { VLUD sample }\end{array}$ \\
\hline $0.1 \%$ & $0.3 \%$ & $0.02 \%$ \\
$1 \%$ & $14.6 \%$ & $1.4 \%$ \\
$5 \%$ & $14.1 \%$ & $17.8 \%$ \\
$10 \%$ & $0.79 \%$ & $28.5 \%$ \\
\hline
\end{tabular}

We tested the probability of obtaining the observed number of close binaries with planets in the UD and VLUD samples for different frequencies of planets using the binomial distribution. This leads to being able to confidently exclude (99\%, see Tables 6 and 7) the preferred value in Pfahl $\&$ Muterspaugh (2006). The observed frequency is marginally compatible only with the upper limit on planet frequency by Pfahl \& Muterspaugh (2006) $(f \sim 1 \%)$ for the UD sample, and hardly compatible for the VLUD sample. The nominal probabilities derived here should be taken with some caution because of the possible incompleteness in binary detection at small separations (anyway estimated to be small, in particular for the VLUD sample, see above) and because the separation distribution is different than the unbiased samples. Nevertheless, our relatively high frequency of planets in close binaries suggests that the dynamical interaction after planet formation is not the unique channel for creating this kind of systems. Instead, we can infer that giant planets might form in binaries that have a small separation at the time of planet formation, possibly in a different way from planets around single stars (and around components of wide binaries).

The run of planet frequency at small separations might shed more light on the formation mechanism(s) of planets in binaries. Desidera \& Barbieri (2007) noted a possible paucity of planets in binaries with a critical semimajor axis for dynamical stability in the range 10-30 AU; only one planet was found in this range, while there are 5 planets with $a_{\text {crit }}$ less than $10 \mathrm{AU}$ and 4 planets with $30<a_{\text {crit }}<50$ AU. A bimodal distribution of planet frequency, with a secondary maximum at $a_{\text {crit }} \sim 3-5 \mathrm{AU}$, is suggested by these data, and it would explain the different characteristics of planets in tight binaries as the result of a different formation mechanism. However, our work is not able to confirm or reject the reality of such a feature, as only 1 star in the UD sample has $10<a_{\text {crit }}<20 \mathrm{AU}$ and 11 have $20<a_{\text {crit }}<30 \mathrm{AU}$ (none of them with planets), making the lack of planet detections insignificant. Furthermore, the closest bin $\left(a_{\text {crit }}<20 \mathrm{AU}\right.$ or periastron $<50 \mathrm{AU}$ ) includes several stars (37 out of 89 ) for which a direct detection of the companion is missing and which are included only on the basis of the astrometric and/or RV trends. Without a determination of the physical parameters of these companions, the study of the run of planet frequency at small separations is not possible.

\section{Conclusions}

After a detailed search for binarity for all stars in the UD sample collected by Fischer \& Valenti (2005), we compared the frequency of planets in binaries and single stars. It turns out that the two frequencies of planets are fairly similar. Even taking possible incompleteness in the binary detection into account in a very conservative way, the frequency of planets in the binaries of the sample cannot be more than a factor of three lower than that of planets orbiting single stars.

For moderately wide binaries, the frequency of planets is independent on separation. Considering the similar mass and period distributions of planets orbiting single stars and components of wide binaries, we then concluded that a wide companion plays a marginal role in the formation and evolution of giant planets.

On the other hand, we found a lower frequency of planets in close binaries than in single stars and components of wide binaries. However, this is probably not as low as required to explain the occurrence of planets in close binaries only as the result of modifications in the binary orbit after planet formation. This, together with the differences in the properties of planets in tight binaries (Desidera \& Barbieri 2007), suggests that planets do form in tight binaries in spite of the unfavorable conditions, possibly in a different way than for planets around single stars. However, crucial issues still need clarification. In fact, it is not yet clear if the run of the planet frequency when moving to smaller separations is characterized by a continuous decrease, by a sharp cut off at which the differences on planet frequency characteristics suddenly onset, or by a bimodal distribution, with a minimum between $10<a_{\text {crit }}<30 \mathrm{AU}$, a relative maximum at $a_{\text {crit }} \sim 3-5 \mathrm{AU}$, a further decrease to zero at extremely small separations, as the zone for dynamical stability of planets vanishes.

These open points might be clarified by a detailed characterization of the binaries in current samples of RV surveys (completeness of binary detection and, when possible, full determination of the orbital elements) and by the completion of dedicated surveys searching for planets in binaries (Desidera et al. 2006; Eggenberger et al. 2006; Konacki 2005).

Acknowledgements. This research has made use of the SIMBAD database, operated at the CDS, Strasbourg, France, of the Washington Double Star Catalog maintained at the US Naval Observatory, and of data products from the Two Micron All Sky Survey. We warmly thank D. Fischer for kindly providing information on the UD sample and S. Lepine for providing the complete tables of his work before publication. We thank the anonymous referee for comments that allowed us to improve the content and the presentation of the paper. We thank R. Gratton for his comments and suggestions. This work was funded by COFIN 2004 "From stars to planets: accretion, disk evolution and planet formation" by the Ministero Univ. e Ricerca Scientifica Italy and by PRIN 2006 "From disk to planetary systems: understanding the origin and demographics of solar and extrasolar planetary systems" by INAF.

\section{References}

Allen, C., Poveda, A., \& Herrera, M. A. 2000, A\&A, 356, 529 Beuzit, J.-L., Ségransan, D., Forveille, T., et al. 2004, A\&A, 425, 997 Boss, A. P. 2006, ApJ, 641, 1148

Burgasser, A. J., Kirkpatrick, J. D., Cutri, R. M., et al. 2000, ApJ, 531, L57

Burgasser, A. J., Kirkpatrick, J. D., \& Lowrance, P. J. 2005, AJ, 129, 2849

Butler, R. P., Vogt, S. S., Marcy, G. W., et al. 2000, ApJ, 545, 504

Butler, R. P., Wright, J. T., Marcy, G. W., et al. 2006, ApJ, 646, 505

Chakraborty, A., Ge, J., \& Debes, J. H. 2002, AJ, 124, 1127

Chauvin, G., Lagrange, A.-M., Udry, S., et al. 2006, A\&A, 456, 1165 Cumming, A. 2004, MNRAS, 354, 1165

Cutri, R. M., Skrutskie, M. F., van Dyk, S., et al. 2003, 2MASS All Sky Catalog of point sources. (The IRSA 2MASS All-Sky Point Source Catalog, NASA/IPAC Infrared Science Archive.

http://irsa.ipac.caltech.edu/applications/Gator/)

Debes, J. H., Ge, J., \& Chakraborty, A. 2002, ApJ, 572, L165

Delfosse, X., Forveille, T., Ségransan, D., et al. 2000, A\&A, 364, 217

Desidera, S., \& Barbieri, M. 2007, A\&A, 462, 345

Desidera, S., Gratton, R., Claudi, R., et al. 2006, in Tenth Anniversary of

51 Peg-b: Status of and prospects for hot Jupiter studies, ed. L. Arnold, F. Bouchy, \& C. Moutou, 119

Desidera, S., Gratton, R. G., Scuderi, S., et al. 2004, A\&A, 420, 683

Dommanget, J., \& Nys, O. 2002, VizieR Online Data Catalog, 1274, 0

Duquennoy, A., \& Mayor, M. 1991, A\&A, 248, 485

Duquennoy, A., Mayor, M., Andersen, J., Carquillat, J. M., \& North, P. 1992, A\&A, 254, L13 
Eggenberger, A., Udry, S., \& Mayor, M. 2004, A\&A, 417, 353

Eggenberger, A., Udry, S., Mayor, M., et al. 2006, in Proc. of ESO Workshop Multiple Stars Across HR Diagram

Els, S. G., Sterzik, M. F., Marchis, F., et al. 2001, A\&A, 370, L1

Fabricius, C., Høg, E., Makarov, V. V., et al. 2002, A\&A, 384, 180

Fischer, D. A., \& Marcy, G. W. 1992, ApJ, 396, 178

Fischer, D. A., Marcy, G. W., Butler, R. P., et al. 2002, PASP, 114, 529

Fischer, D. A., \& Valenti, J. 2005, ApJ, 622, 1102

Fisher, G. F., Hall, D. S., Henry, G. W., et al. 1983, Informational Bulletin on Variable Stars, 2259, 1

Girardi, L., Bertelli, G., Bressan, A., et al. 2002, A\&A, 391, 195

Gizis, J. E., Kirkpatrick, J. D., Burgasser, A., et al. 2001, ApJ, 551, L163

Gizis, J. E., Monet, D. G., Reid, I. N., Kirkpatrick, J. D., \& Burgasser, A. J. 2000, MNRAS, 311, 385

Gliese, W., \& Jahreiß, H. 1991, Preliminary Version of the Third Catalogue of Nearby Stars, Tech. rep.

Golimowski, D. A., Henry, T. J., Krist, J. E., et al. 2000, AJ, 120, 2082

Gould, A., \& Chanamé, J. 2004, ApJS, 150, 455

Gratton, R. G., Bonanno, G., Claudi, R. U., et al. 2001, A\&A, 377, 123

Griffin, R. F., \& Gunn, J. E. 1981, AJ, 86, 588

Halbwachs, J. L. 1986, Bulletin d'Information du Centre de Donées Stellaires, 30,129

Halbwachs, J. L., Arenou, F., Mayor, M., Udry, S., \& Queloz, D. 2000, A\&A, 355,581

Hale, A. 1994, AJ, 107, 306

Hartkopf, W. I., \& Mason, B. D. 2006, Sixth Catalog of Orbits of Visual Binary Stars, http: //ad.usno.navy.mil/wds/orb6.html

Heintz, W. D. 1994, AJ, 108, 2338

Holman, M. J., \& Wiegert, P. A. 1999, AJ, 117, 621

Huensch, M., Schmitt, J. H. M. M., \& Voges, W. 1998, A\&AS, 132, 155

Jones, H. R. A., Butler, P., Marcy, G. W., et al. 2002, MNRAS, 337, 1170

Jones, H. R. A., Butler, R. P., Tinney, C. G., et al. 2006, MNRAS, 369, 249

Kley, W. 2000, in IAU Symposium, 211P

Konacki, M. 2005, ApJ, 626, 431

Lagrange, A.-M., Beust, H., Udry, S., Chauvin, G., \& Mayor, M. 2006, A\&A, 459,955

Latham, D. W., Stefanik, R. P., Torres, G., et al. 2002, AJ, 124, 1144

Lépine, S., \& Bongiorno, B. 2007, AJ, 133, 889

Linsky, J. L. 1984, NASA STI/Recon Technical Report N, 85, 13704

Liu, M. C., Fischer, D. A., Graham, J. R., et al. 2002, ApJ, 571, 519

Luhman, K. L., Patten, B. M., Marengo, M., et al. 2006

Luhman, K. L., Patten, B. M., Marengo, M., et al. 2007, ApJ, 654, 570

Makarov, V. V., \& Kaplan, G. H. 2005, AJ, 129, 2420

Marcy, G. W., Butler, R. P., Williams, E., et al. 1997, ApJ, 481, 926
Marcy, G., Butler, R. P., Fischer, D., et al. 2005, Progress of Theoretical Physics Supplement, 158, 24

Martín, E. L., Basri, G., Pavlenko, Y., \& Lyubchik, Y. 2002, ApJ, 579, 437

Mayor, M., \& Queloz, D. 1995, Nature, 378, 355

Metchev, S. 2006, Ph.D. Thesis

Mugrauer, M., \& Neuhäuser, R. 2005, MNRAS, 361, L15

Mugrauer, M., Neuhäuser, R., Guenther, E. W., et al. 2004, A\&A, 417, 1031

Nidever, D. L., Marcy, G. W., Butler, R. P., Fischer, D. A., \& Vogt, S. S. 2002, ApJS, 141, 503

Nordström, B., Mayor, M., Andersen, J., et al. 2004, A\&A, 418, 989

Perrier, C., Sivan, J.-P., Naef, D., et al. 2003, A\&A, 410, 1039

Perryman, M. A. C., \& ESA. 1997, The HIPPARCOS and TYCHO catalogues. Astrometric and photometric star catalogues derived from the ESA HIPPARCOS Space Astrometry Mission, The Hipparcos and Tycho catalogues. Astrometric and photometric star catalogues derived from the ESA Hipparcos Space Astrometry Mission, Publisher: Noordwijk (Netherlands: ESA Publications Division), Series: ESA SP Ser. Vol No: 1200

Pfahl, E., \& Muterspaugh, M. 2006, ApJ, 652, 1694

Pichardo, B., Sparke, L. S., \& Aguilar, L. A. 2005, MNRAS, 359, 521

Pinfield, D. J., Jones, H. R. A., Lucas, P. W., et al. 2006, MNRAS, 368, 1281

Pourbaix, D., Tokovinin, A. A., Batten, A. H., et al. 2004, A\&A, 424, 727

Quist, C. F., \& Lindegren, L. 2000, A\&A, 361, 770

Raghavan, D., Henry, T. J., Mason, B. D., et al. 2006, ApJ, 646, 523

Reffert, S., \& Quirrenbach, A. 2006, A\&A, 449, 699

Reid, I. N., \& Gizis, J. E. 1997, AJ, 113, 2246

Salim, S., \& Gould, A. 2003, ApJ, 582, 1011

Thébault, P., Marzari, F., \& Scholl, H. 2006, Icarus, 183, 193

Thévenin, F., Kervella, P., Pichon, B., et al. 2005, A\&A, 436, 253

Tinney, C. G., Butler, R. P., Marcy, G. W., et al. 2003, ApJ, 587, 423

Tokovinin, A. A. 1997, A\&AS, 124, 75

Tokovinin, A. A., Griffin, R. F., Balega, Y. Y., Pluzhnik, E. A., \& Udry, S. 2000, Astron. Lett., 26, 116

Turner, N. H., ten Brummelaar, T. A., McAlister, H. A., et al. 2001, AJ, 121, 3254

Valenti, J. A., \& Fischer, D. A. 2005, ApJS, 159, 141

Vogt, S. S., Butler, R. P., Marcy, G. W., et al. 2002, ApJ, 568, 352

Wittenmyer, R. A., Endl, M., Cochran, W. D., et al. 2006, AJ, 132, 177

Worley, C. E. 1967a, AJ, 72, 899

Worley, C. E. 1967b, in On the Evolution of Double Stars, ed. J. Dommanget, 221

Worley, C. E., \& Douglass, G. G. 1997, A\&AS, 125, 523

Worley, C. E., \& Heintz, W. D. 1983, Publications of the US Naval Observatory Second Series, 24, 1

Wright, J. T., Marcy, G. W., Butler, R. P., \& Vogt, S. S. 2004, ApJS, 152, 261

Zucker, S., \& Mazeh, T. 2001, ApJ, 562, 549 
M. Bonavita and S. Desidera: The frequency of planets in multiple systems, Online Material $p 1$

\section{Online Material}


M. Bonavita and S. Desidera: The frequency of planets in multiple systems, Online Material p 2

Table 8. Properties of binaries found in the UD sample: projected separation ( $\operatorname{arcsec}$ ), eccentricity and semimajor axis (when available), masses of the object and the companion, and critical semimajor axis for dynamical stability of planets (Holman \& Wiegert 1999). For systems for which only the projected separation was available (empty spaces in eccentricity column) the semimajor axis was derived from the projected separation using the relation $a(\mathrm{AU})=1.31 \rho(\operatorname{arcsec}) d(\mathrm{pc})$ (see Fischer et al. 2002; Duquennoy \& Mayor 1991). The asterisk in the last column marks systems discussed individually in Appendix A. The mass flag indicates the source for the companion mass: a: $M_{\text {comp }}$ from VF06; b: $M_{\text {comp }}$ from Reid \& Gizis (1997); Delfosse et al. (2000); c: $M_{\text {comp }}$ from individual papers (see Reference below).

\begin{tabular}{|c|c|c|c|c|c|c|c|c|}
\hline HD & $\begin{array}{c}\rho \\
(\operatorname{arcsec})\end{array}$ & ecc & $\begin{array}{c}a \\
(\mathrm{AU})\end{array}$ & $\begin{array}{l}a_{\text {crit }} \\
(\mathrm{AU})\end{array}$ & $\begin{array}{l}M_{\text {obj }} \\
\left(M_{\odot}\right) \\
\end{array}$ & $\begin{array}{l}M_{\text {com }} \\
\left(M_{\odot}\right)\end{array}$ & Mass Flag & Remarks \\
\hline $531 \mathrm{~A}$ & 5.30 & & 482.30 & 76.37 & 1.64 & 1.66 & $\mathrm{a}$ & $\mathrm{S}$ \\
\hline $531 \mathrm{~B}$ & 5.30 & & 482.30 & 76.94 & 1.66 & 1.64 & a & S \\
\hline 3074 & 4.80 & & 225.89 & 38.01 & 1.20 & 0.99 & $\mathrm{~b}$ & S \\
\hline 3651 & 43.20 & & 623.38 & 151.00 & 0.89 & 0.06 & $\mathrm{~b}$ & $S(*)$ \\
\hline 3770 & & & & & 1.25 & & & S, RV \\
\hline 3795 & & & & & 1.94 & & & $\mathrm{~S}, \mathrm{RV}, \Delta \mu$ \\
\hline 3821 & 8.45 & & 284.51 & 51.50 & 1.00 & 0.63 & b & $\mathrm{S}$ \\
\hline 4614 & 12.49 & 0.49 & 72.00 & 10.30 & 0.99 & 0.51 & b & S \\
\hline 4747 & & 0.64 & 6.70 & 0.79 & 0.82 & 0.04 & $\mathrm{c}$ & S, SB \\
\hline 6734 & & & & & 1.08 & & & $\mathrm{~S}, \Delta \mu$ \\
\hline 6872 A & 14.60 & & 1442.48 & 271.29 & 1.91 & 1.03 & b & $\mathrm{S}$ \\
\hline 6872 B & 14.60 & & 1442.48 & 187.22 & 1.03 & 1.91 & a & S \\
\hline 7693 & 0.90 & 0.04 & 23.40 & 5.98 & 0.84 & 0.89 & b & $\mathrm{S}(*)$ \\
\hline 8765 & & & & & 1.20 & & & $\mathrm{~S}, \Delta \mu, \mathrm{G}$ \\
\hline 10360 & 11.20 & 0.53 & 52.20 & 5.66 & 0.75 & 0.77 & a & $\mathrm{S}$ \\
\hline 10361 & 11.20 & 0.53 & 52.20 & 5.74 & 0.77 & 0.75 & $\mathrm{a}$ & $\mathrm{S}$ \\
\hline 11964 & 40.50 & & 1790.10 & 325.00 & 1.13 & 0.67 & b & $\mathrm{S}(*)$ \\
\hline 13043 & 79.20 & & 3809.52 & 684.35 & 1.14 & 0.74 & b & $\mathrm{S}$ \\
\hline 13507 & & 0.14 & 4.30 & 1.57 & 1.00 & 0.05 & $\mathrm{c}$ & $\mathrm{S}, \mathrm{SB}, \Delta \mu\left(^{*}\right)$ \\
\hline 13531 & 0.70 & & 23.30 & 5.21 & 0.94 & 0.19 & c & S \\
\hline $13612 \mathrm{~B}$ & 16.70 & & 1000.83 & 121.14 & 1.02 & 2.32 & $\mathrm{c}$ & $S(*)$ \\
\hline 16141 & 6.20 & & 289.35 & 62.00 & 1.15 & 0.29 & b & $S(*)$ \\
\hline 16160 & 3.30 & 0.75 & 15.00 & 1.04 & 0.76 & 0.09 & $\mathrm{c}$ & $S(*)$ \\
\hline 17037 & & & & & 1.23 & & & S, RV \\
\hline 16895 & 20.50 & 0.13 & 249.50 & 76.20 & 1.24 & 0.43 & b & S \\
\hline 18143 & 44.10 & & 1312.86 & 222.85 & 0.90 & 0.72 & b & $\mathrm{S}(*)$ \\
\hline 18445 & 0.10 & 0.56 & 1.06 & 0.14 & 0.78 & 0.18 & $\mathrm{c}$ & $\mathrm{S}(*)$ \\
\hline 20766 & 310.00 & & 4876.30 & 711.69 & 0.91 & 1.19 & $\mathrm{a}$ & S \\
\hline 20807 & 310.00 & & 4876.30 & 736.00 & 0.95 & 1.12 & $\mathrm{a}$ & S \\
\hline 21019 & 3.90 & & 187.08 & 36.48 & 1.11 & 0.51 & b & $\mathrm{S}$ \\
\hline 23439 & 8.00 & & 254.80 & 37.41 & 0.67 & 0.86 & $\mathrm{c}$ & $\mathrm{S}(*)$ \\
\hline 26491 & & & & & 1.00 & & & $\mathrm{~S}, \Delta \mu$ \\
\hline $28255 \mathrm{~A}$ & 5.90 & & 199.42 & 31.78 & 1.07 & 1.06 & $\mathrm{a}$ & $\mathrm{S}$ \\
\hline 28255 B & 5.90 & & 199.42 & 31.60 & 1.06 & 1.07 & $\mathrm{a}$ & S \\
\hline 29461 & & & & & 1.20 & & & S, RV \\
\hline 29836 & 100.00 & & 5590.00 & 852.14 & 1.19 & 1.36 & $\mathrm{c}$ & $S(*)$ \\
\hline 30339 & & 0.25 & 0.13 & 0.04 & 1.39 & 0.07 & $\mathrm{c}$ & $\mathrm{S}, \mathrm{SB}$ \\
\hline 30649 & & & & & 0.90 & & & $\mathrm{~S}, \mathrm{RV}(*)$ \\
\hline 31412 & & & & & 1.17 & & & $\mathrm{~S}, \mathrm{RV}(*)$ \\
\hline 32923 & 0.18 & 0.90 & 2.86 & 0.03 & 1.03 & 1.11 & b & S \\
\hline 33473 & 100.00 & & 7475.00 & 1358.02 & 1.32 & 0.82 & b & S \\
\hline 35956 & & 0.62 & 2.60 & 0.30 & 0.98 & 0.18 & c & S, SB $(*)$ \\
\hline 37394 & 97.50 & & 1546.35 & 292.41 & 0.93 & 0.49 & b & $\mathrm{S}$ \\
\hline 39587 & & 0.45 & 5.90 & 1.13 & 1.05 & 0.14 & $\mathrm{c}$ & S, SB \\
\hline 40397 & 4.10 & & 123.66 & 25.62 & 0.92 & 0.31 & b & $\mathrm{S}$ \\
\hline 43587 & & 0.80 & 11.60 & 0.53 & 1.06 & 0.34 & $\mathrm{c}$ & S, SB $(*)$ \\
\hline 44120 & 40.40 & & 1911.73 & 330.66 & 1.23 & 0.92 & $\mathrm{~b}$ & S \\
\hline 45701 & & & & & 1.18 & & & $\mathrm{~S}, \Delta \mu$ \\
\hline 45588 & 41.20 & & 1601.44 & 325.89 & 1.21 & 0.45 & b & $S$ \\
\hline 47157 & 10.10 & & 502.88 & 105.72 & 1.13 & 0.35 & $\mathrm{~b}$ & S \\
\hline 50281 & 58.30 & & 659.37 & 118.04 & 0.76 & 0.50 & $\mathrm{~b}$ & $\mathrm{~S}$ \\
\hline 50639 & & & & & 1.16 & & & $\mathrm{~S}, \mathrm{RV}, \Delta \mu$ \\
\hline 51929 & & & & & 0.86 & & & $\mathrm{~S}, \Delta \mu$ \\
\hline 53705 & 21.00 & & 425.88 & 69.47 & 0.97 & 0.89 & $\mathrm{a}$ & $\mathrm{S}(*)$ \\
\hline 53706 & 21.00 & & 425.88 & 65.90 & 0.89 & 0.97 & $\mathrm{a}$ & $\mathrm{S}(*)$ \\
\hline 61606 & 58.10 & & 1072.53 & 184.33 & 0.81 & 0.62 & $\mathrm{~b}$ & S \\
\hline 63754 & 5.60 & & 376.38 & 71.81 & 1.50 & 0.76 & b & S \\
\hline 64468 & & 0.26 & 0.56 & 0.15 & 0.81 & 0.14 & c & S, SB \\
\hline 65907 & 60.00 & & 1263.60 & 201.45 & 0.99 & 0.98 & $\mathrm{c}$ & $S(*)$ \\
\hline 65277 & 4.10 & & 93.27 & 18.82 & 0.72 & 0.28 & b & $\mathrm{S}, \Delta \mu$ \\
\hline 65430 & & 0.32 & 4.00 & 1.05 & 0.83 & 0.06 & $\mathrm{c}$ & S, SB \\
\hline
\end{tabular}


M. Bonavita and S. Desidera: The frequency of planets in multiple systems, Online Material p 3

Table 8. continued.

\begin{tabular}{|c|c|c|c|c|c|c|c|c|}
\hline$\overline{\mathrm{HD}}$ & $\begin{array}{c}\rho \\
(\operatorname{arcsec})\end{array}$ & ecc & $\begin{array}{c}\mathrm{a} \\
(\mathrm{AU})\end{array}$ & $\begin{array}{c}a_{\text {crit }} \\
\text { (AU) }\end{array}$ & $\begin{array}{l}M_{o b j} \\
\left(M_{\odot}\right)\end{array}$ & $\begin{array}{l}M_{\text {com }} \\
\left(M_{\odot}\right)\end{array}$ & Mass Flag & $\overline{\overline{\text { Remarks }}}$ \\
\hline 66171 & 49.00 & & 3013.01 & 730.27 & 0.91 & 0.07 & $\mathrm{~b}$ & $\bar{S}$ \\
\hline 72760 & 0.90 & & 25.51 & 5.92 & 0.91 & 0.13 & $\mathrm{c}$ & $\mathrm{S}, \Delta \mu(*)$ \\
\hline 72780 & & & & & 1.28 & & & $S, R V$ \\
\hline 73668 & 26.50 & & 1229.86 & 210.35 & 1.13 & 0.88 & $\mathrm{~b}$ & $\mathrm{~S}$ \\
\hline 77407 & 1.60 & & 50.00 & 9.65 & 1.12 & 0.54 & $\mathrm{c}$ & $S(*)$ \\
\hline 86728 & 134.00 & & 2595.58 & 626.32 & 1.08 & 0.09 & b & $S(*)$ \\
\hline 88218 & 2.00 & & 79.82 & 14.49 & 1.09 & 0.68 & b & $\mathrm{S}$ \\
\hline 90839 & 122.80 & & 2043.39 & 372.01 & 1.12 & 0.69 & b & $S(*)$ \\
\hline 92222 & 17.70 & & 2070.90 & 332.90 & 1.09 & 1.05 & $\mathrm{c}$ & $\mathrm{S}(*)$ \\
\hline 92987 & & & & & 1.15 & & & $\mathrm{~S}, \Delta \mu$ \\
\hline HI 52942 & 17.60 & & 2779.92 & 418.07 & 1.04 & 1.24 & b & $S(*)$ \\
\hline HI 52940 & & 0.37 & 2.60 & 0.60 & 1.12 & 0.12 & $\mathrm{c}$ & S, SB (*) \\
\hline 97334 & 90.00 & & 2620.00 & 649.08 & 1.09 & 0.05 & $\mathrm{c}$ & $\mathrm{S}(*)$ \\
\hline 99491 & 28.80 & & 666.43 & 111.12 & 1.01 & 0.86 & a & $\mathrm{S}$ \\
\hline 99492 & 28.80 & & 666.43 & 100.71 & 0.86 & 1.01 & a & $S(*)$ \\
\hline 100180 & 15.40 & & 460.46 & 82.24 & 1.10 & 0.73 & b & $\mathrm{S}$ \\
\hline 100623 & 17.00 & & 209.95 & 51.31 & 0.77 & 0.05 & b & $\mathrm{S}$ \\
\hline 101177 & 9.70 & & 293.81 & 45.85 & 0.99 & 1.05 & $\mathrm{c}$ & $S(*)$ \\
\hline 102365 & 23.00 & & 275.08 & 65.42 & 0.86 & 0.09 & b & $\mathrm{S}$ \\
\hline 103432 & 73.20 & & 3520.92 & 565.26 & 0.92 & 0.89 & b & $\mathrm{S}$ \\
\hline 103829 & & & & & 1.20 & & & S, RV \\
\hline 104556 B & & & & & 1.12 & & & $\mathrm{~S}, \Delta \mu, \mathrm{G}$ \\
\hline 105113 & 6.60 & & 440.15 & 75.43 & 1.28 & 0.99 & b & $\mathrm{S}$ \\
\hline 107705 & 20.00 & & 774.80 & 141.13 & 1.22 & 0.75 & b & S \\
\hline 111031 & & & & & 1.14 & & & $\mathrm{~S}, \Delta \mu$ \\
\hline 111398 & & & 75.00 & 17.52 & 1.06 & 0.14 & b & S \\
\hline 111484 A & 8.80 & & 846.56 & 134.25 & 1.38 & 1.39 & $\mathrm{a}$ & $\mathrm{S}$ \\
\hline 111484 B & 8.80 & & 846.56 & 134.84 & 1.39 & 1.38 & $\mathrm{a}$ & $\mathrm{S}$ \\
\hline 114729 & 8.00 & & 364.00 & 79.00 & 1.00 & 0.25 & b & $S(*)$ \\
\hline 116442 & 26.50 & & 564.98 & 90.90 & 0.76 & 0.73 & a & $\mathrm{S}$ \\
\hline 116443 & 26.50 & & 564.98 & 88.68 & 0.73 & 0.76 & $\mathrm{a}$ & $\mathrm{S}$ \\
\hline 120066 & 488.50 & & 19432.53 & 3581.91 & 1.16 & 0.68 & b & $\mathrm{S}$ \\
\hline 120237 & 11.60 & & 426.76 & 81.04 & 1.16 & 0.60 & b & $\mathrm{S}$ \\
\hline 120476 & 3.40 & 0.44 & 33.15 & 4.36 & 0.76 & 0.83 & b & $\mathrm{S}$ \\
\hline 120690 & & & & & 1.02 & & & $\mathrm{~S}, \Delta \mu$ \\
\hline 120780 & & & & & 0.74 & & & $\mathrm{~S}, \mathrm{G}, \Delta \mu(*)$ \\
\hline 121384 & 33.00 & & 1634.49 & 328.24 & 1.18 & 0.47 & b & S \\
\hline 122742 & & 0.48 & 5.30 & 0.75 & 0.92 & 0.54 & $\mathrm{c}$ & S, SB \\
\hline 125455 & 15.30 & & 413.71 & 91.76 & 0.79 & 0.17 & b & $\mathrm{S}$ \\
\hline 126614 & 41.90 & & 3725.75 & 883.39 & 1.19 & 0.13 & b & S \\
\hline 128428 & 0.80 & & 54.39 & 9.99 & 1.26 & 0.75 & b & $\mathrm{S}$ \\
\hline 128621 & 17.51 & 0.51 & 22.76 & 2.44 & 0.89 & 1.12 & a & $S(*)$ \\
\hline 128620 & 17.51 & 0.51 & 22.76 & 2.79 & 1.12 & 0.89 & $\mathrm{a}$ & $\mathrm{S}(*)$ \\
\hline 128674 & 490.00 & & 17453.80 & 2906.33 & 0.83 & 0.71 & b & $\mathrm{S}$ \\
\hline 129814 & & & & & 1.06 & & & $\mathrm{~S}, \mathrm{RV}, \Delta \mu$ \\
\hline 131156 & 4.90 & 0.51 & 32.80 & 3.93 & 0.92 & 0.79 & b & $S(*)$ \\
\hline 131511 & & 0.51 & 0.52 & 0.07 & 0.93 & 0.45 & c & S, SB \\
\hline 131977 & 24.90 & & 190.98 & 28.29 & 0.76 & 0.95 & $\mathrm{c}$ & $S(*)$ \\
\hline 131923 & & & & & 1.04 & & & $\mathrm{~S}, \Delta \mu, \mathrm{G}$ \\
\hline 133161 & & & & & 1.18 & & & $\mathrm{~S}, \Delta \mu$ \\
\hline 134440 & 302.00 & & 11581.70 & 1830.52 & 0.55 & 0.56 & $\mathrm{a}$ & $\mathrm{S}$ \\
\hline 134439 & 302.00 & & 11581.70 & 1850.83 & 0.56 & 0.55 & $\mathrm{a}$ & S \\
\hline 134331 & 50.60 & & 1993.13 & 325.83 & 1.12 & 1.02 & $\mathrm{a}$ & S \\
\hline 134330 & 50.60 & & 1993.13 & 307.70 & 1.02 & 1.12 & a & $\mathrm{S}$ \\
\hline 135101 & 23.50 & & 870.67 & 209.00 & 1.07 & 0.92 & $\mathrm{c}$ & S \\
\hline 136580 & & & & & 1.17 & & & $\mathrm{~S}, \mathrm{RV}, \Delta \mu$ \\
\hline 137778 & 51.90 & & 1403.38 & 182.11 & 0.90 & 1.67 & c & $S(*)$ \\
\hline 139323 & 121.90 & & 3533.88 & 468.58 & 0.89 & 1.55 & c & $\mathrm{S}(*)$ \\
\hline 139477 & 42.00 & & 1042.86 & 221.21 & 0.75 & 0.22 & b & S \\
\hline 140913 & & 0.54 & 0.55 & 0.09 & 1.17 & 0.04 & $\mathrm{c}$ & S, SB \\
\hline 140901 & 8.10 & & 160.06 & 36.49 & 1.00 & 0.17 & b & $\mathrm{S}$ \\
\hline 142229 & & & & & 1.09 & & & S, RV \\
\hline 144579 & 70.10 & & 1312.27 & 308.93 & 0.75 & 0.09 & $\mathrm{~b}$ & $\mathrm{~S}$ \\
\hline 145435 & & & & & 1.19 & & & $\mathrm{~S}, \Delta \mu$ \\
\hline $145958 \mathrm{~A}$ & 4.20 & 0.39 & 124.00 & 18.66 & 0.90 & 0.89 & $\mathrm{a}$ & $\mathrm{S}(*)$ \\
\hline 145958 B & 4.20 & 0.39 & 124.00 & 18.53 & 0.89 & 0.90 & a & $\mathrm{S}$ \\
\hline
\end{tabular}


M. Bonavita and S. Desidera: The frequency of planets in multiple systems, Online Material p 4

Table 8. continued.

\begin{tabular}{|c|c|c|c|c|c|c|c|c|}
\hline$\overline{\mathrm{HD}}$ & $\begin{array}{c}\rho \\
(\operatorname{arcsec})\end{array}$ & ecc & $\begin{array}{c}\mathrm{a} \\
(\mathrm{AU})\end{array}$ & $\begin{array}{l}a_{\text {crit }} \\
\text { (AU) }\end{array}$ & $\begin{array}{l}M_{o b j} \\
\left(M_{\odot}\right)\end{array}$ & $\begin{array}{l}M_{\text {com }} \\
\left(M_{\odot}\right)\end{array}$ & 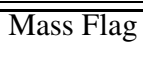 & $\overline{\overline{\text { Remarks }}}$ \\
\hline 146362 & 6.80 & 0.76 & 130.00 & 5.03 & 1.12 & 2.19 & $c$ & $S(*)$ \\
\hline 147722 & 5.40 & & 220.43 & 33.89 & 1.16 & 1.29 & a & $\mathrm{S}$ \\
\hline 147723 & 5.40 & & 220.43 & 36.17 & 1.29 & 1.16 & $\mathrm{a}$ & $\mathrm{S}$ \\
\hline 149806 & 5.90 & & 154.17 & 31.20 & 0.94 & 0.36 & $\mathrm{~b}$ & $\mathrm{~S}$ \\
\hline 150554 & 11.60 & & 675.58 & 138.12 & 1.13 & 0.41 & $\mathrm{c}$ & $\mathrm{S}$ \\
\hline 150248 & & & & & 0.96 & & & $\mathrm{~S}, \Delta \mu$ \\
\hline 151090 & 163.60 & & 10102.30 & 1929.65 & 1.17 & 0.59 & $\mathrm{~b}$ & $\mathrm{~S}$ \\
\hline 156274 & 8.65 & 0.78 & 91.65 & 4.30 & 0.79 & 0.47 & $\mathrm{~b}$ & $\mathrm{~S}$ \\
\hline 157466 & & & & & 0.92 & & & $\mathrm{~S}, \Delta \mu$ \\
\hline 159909 & & & & & 1.04 & & b & S \\
\hline 161797 & 1.42 & 0.32 & 22.00 & 3.90 & 1.15 & 0.13 & $\mathrm{c}$ & $\mathrm{S}, \mathrm{RV}, \Delta \mu(*)$ \\
\hline 164595 & 88.00 & & 3306.16 & 674.36 & 0.98 & 0.36 & b & $\mathrm{S}$ \\
\hline 166553 & 1.40 & & 77.17 & 13.83 & 1.22 & 0.80 & b & $\mathrm{S}$ \\
\hline 167215 & & & & & 1.15 & & & $\mathrm{~S}, \mathrm{G}, \Delta \mu\left(^{*}\right)$ \\
\hline 167665 & & & & & 1.11 & & & S, RV \\
\hline 169586 & & & & & 1.29 & & & $\mathrm{~S}, \Delta \mu$ \\
\hline 169822 & & 0.48 & 0.84 & 0.13 & 0.91 & 0.30 & $\mathrm{c}$ & S, SB (*) \\
\hline 173667 & 48.20 & & 1196.81 & 266.63 & 1.54 & 0.32 & b & $\mathrm{S}$ \\
\hline 174457 & & 0.23 & 1.90 & 0.60 & 1.07 & 0.06 & $\mathrm{c}$ & S, SB \\
\hline 175345 & 5.40 & & 348.89 & 67.43 & 1.17 & 0.56 & b & $\mathrm{S}$ \\
\hline 179957 & 8.20 & & 254.77 & 40.25 & 1.01 & 1.03 & $\mathrm{a}$ & $\mathrm{S}$ \\
\hline 179958 & 8.20 & & 254.77 & 40.73 & 1.03 & 1.01 & $\mathrm{a}$ & $\mathrm{S}$ \\
\hline 179140 & 0.50 & & 34.06 & 5.60 & 1.12 & 1.00 & $\mathrm{~b}$ & $\mathrm{~S}$ \\
\hline 184860 & & 0.67 & 1.40 & 0.15 & 0.77 & 0.03 & $\mathrm{c}$ & $\mathrm{S}, \mathrm{SB}(*)$ \\
\hline 185395 & 37.00 & & 894.66 & 196.56 & 1.34 & 0.31 & b & $\mathrm{S}$ \\
\hline 187691 & 14.40 & & 363.17 & 79.35 & 1.37 & 0.33 & b & $S(*)$ \\
\hline 190360 & 188.60 & & 3898.36 & 864.00 & 1.01 & 0.20 & b & $S(*)$ \\
\hline 190406 & 0.80 & & 18.41 & 4.52 & 1.09 & 0.065 & $\mathrm{c}$ & $\mathrm{S}, \mathrm{RV}(*)$ \\
\hline 190067 & 2.86 & & 55.00 & 12.91 & 0.80 & 0.10 & $\mathrm{~b}$ & $\mathrm{~S}(*)$ \\
\hline 190771 & & & & & 1.07 & & & $\mathrm{~S}, \mathrm{RV}, \Delta \mu$ \\
\hline 191785 & 103.80 & & 2766.27 & 559.07 & 0.83 & 0.32 & b & $\mathrm{S}$ \\
\hline 191408 & 7.10 & & 56.30 & 11.60 & 0.69 & 0.24 & b & $\mathrm{S}$ \\
\hline 192343 & 43.40 & & 3627.81 & 577.95 & 1.28 & 1.27 & b & $\mathrm{S}$ \\
\hline 192344 & 43.40 & & 3627.81 & 575.18 & 1.27 & 1.28 & b & S \\
\hline 194766 B & 43.60 & & 2635.62 & 423.63 & 1.10 & 1.06 & b & $\mathrm{S}$ \\
\hline 195564 & 2.90 & & 91.23 & 17.39 & 1.12 & 0.57 & b & $\mathrm{S}$ \\
\hline 196201 & 2.20 & & 109.54 & 18.72 & 0.87 & 0.68 & b & S \\
\hline 197076 & 125.00 & & 3412.50 & 767.57 & 0.99 & 0.19 & b & $\mathrm{S}(*)$ \\
\hline 196068 & 17.40 & & 882.18 & 159.88 & 1.69 & 1.06 & b & $\mathrm{S}$ \\
\hline 196885 & 0.70 & & 30.03 & 5.80 & 1.25 & 0.60 & $\mathrm{c}$ & $S,(*)$ \\
\hline 198387 & & & & & 1.32 & & & $\mathrm{~S}, \mathrm{G}, \Delta \mu(*)$ \\
\hline 199598 & & & & & 1.15 & & & $\mathrm{~S}, \Delta \mu$ \\
\hline 200565 & & & & & 1.06 & & & $\mathrm{~S}, \mathrm{RV}, \Delta \mu$ \\
\hline 206387 & 3.70 & & 264.07 & 48.40 & 1.20 & 0.72 & b & $\mathrm{S}$ \\
\hline 206860 & 43.20 & & 1033.34 & 260.95 & 1.07 & 0.021 & $\mathrm{c}$ & $\mathrm{S}$ \\
\hline 208776 & & 0.27 & 4.20 & 0.97 & 1.14 & 0.51 & $\mathrm{c}$ & S, SB \\
\hline 212330 & 81.10 & & 2161.31 & 513.18 & 1.12 & 0.12 & $\mathrm{~b}$ & $S$ \\
\hline 212168 & 20.10 & & 603.60 & 112.67 & 1.06 & 0.59 & b & $\mathrm{S}$ \\
\hline 213519 & 62.00 & & 3481.92 & 734.00 & 1.05 & 0.32 & b & $\mathrm{S}$ \\
\hline 214953 & 7.80 & & 239.30 & 44.82 & 1.13 & 0.62 & $\mathrm{~b}$ & $S$ \\
\hline 215578 & & & & & 1.02 & & & S, RV (*) \\
\hline 215648 & 11.80 & & 2485.08 & 538.88 & 1.26 & 0.32 & b & $\mathrm{S}$ \\
\hline 217004 & 8.90 & & 794.86 & 136.69 & 1.27 & 0.97 & b & S \\
\hline 218101 & & & & & 1.26 & & & $\mathrm{~S}, \Delta \mu$ \\
\hline $219542 \mathrm{~A}$ & 5.28 & & 388.00 & 71.00 & 1.08 & 1.05 & $\mathrm{c}$ & $\mathrm{S}(*)$ \\
\hline 219542 B & 5.28 & & 388.00 & 67.00 & 1.05 & 1.08 & $\mathrm{c}$ & $\mathrm{S}(*)$ \\
\hline 219834 & 13.00 & & 331.24 & 48.80 & 0.74 & 0.94 & $\mathrm{c}$ & $\mathrm{S}(*)$ \\
\hline 220077 & 0.20 & & 19.89 & 3.19 & 1.09 & 1.06 & b & $\mathrm{S}$ \\
\hline 221830 & 8.00 & & 335.92 & 70.79 & 0.95 & 0.29 & $\mathrm{~b}$ & $\mathrm{~S}$ \\
\hline 223084 & & & & & 1.09 & & & $\mathrm{~S}, \mathrm{RV}, \Delta \mu$ \\
\hline \multicolumn{9}{|c|}{ Stars with planets } \\
\hline 142 & 5.40 & & 179.71 & 35.00 & 1.24 & 0.56 & $\mathrm{~b}$ & $\bar{P}$ \\
\hline 9826 & 55.50 & & 974.02 & 223.00 & 1.32 & 0.19 & $\mathrm{~b}$ & $\mathrm{P}$ \\
\hline 13445 & 1.30 & 0.40 & 18.40 & 3.10 & 0.77 & 0.49 & $\mathrm{c}$ & $\mathrm{P}, \Delta \mu(*)$ \\
\hline 20782 & 252.20 & & 11802.96 & 1940.00 & 1.00 & 0.84 & $\mathrm{c}$ & $\mathrm{P}(*)$ \\
\hline 27442 & 13.80 & & 326.51 & 62.00 & 1.49 & 0.60 & $\mathrm{c}$ & $\mathrm{P}(*)$ \\
\hline
\end{tabular}


Table 8. continued.

\begin{tabular}{|c|c|c|c|c|c|c|c|c|}
\hline$\overline{\mathrm{HD}}$ & $\begin{array}{c}\rho \\
(\operatorname{arcsec})\end{array}$ & ecc & $\begin{array}{c}\mathrm{a} \\
(\mathrm{AU})\end{array}$ & $\begin{array}{c}a_{\text {crit }} \\
\text { (AU) }\end{array}$ & $\begin{array}{l}M_{o b j} \\
\left(M_{\odot}\right)\end{array}$ & $\begin{array}{l}M_{\text {com }} \\
\left(M_{\odot}\right)\end{array}$ & $\overline{\text { Mass Flag }}$ & $\overline{\overline{\text { Remarks }}}$ \\
\hline 38529 & 283.02 & & 15600.10 & 3190.00 & 1.47 & 0.50 & $\mathrm{~b}$ & $\mathrm{P}, \mathrm{G}(*)$ \\
\hline 40979 & 192.20 & & 8320.34 & 1488.00 & 1.19 & 0.75 & b & $P$ \\
\hline 46375 & 10.30 & & 447.23 & 80.00 & 0.92 & 0.60 & b & $\mathrm{P}$ \\
\hline 75732 & 84.90 & & 1379.63 & 291.00 & 0.91 & 0.26 & b & $\mathrm{P}$ \\
\hline 120136 & 12.00 & 0.91 & 245.00 & 2.80 & 1.35 & 0.40 & b & $\mathrm{P}(*)$ \\
\hline $178911 \mathrm{~B}$ & 13.60 & & 830.96 & 108.00 & 1.42 & 1.89 & $\mathrm{c}$ & $\mathrm{P}(*)$ \\
\hline 188015 & 13.00 & & 888.94 & 198.00 & 1.25 & 0.21 & b & $\mathrm{P}$ \\
\hline 195019 & 4.00 & & 194.48 & 35.00 & 1.07 & 0.70 & b & $\mathrm{P}$ \\
\hline 196050 & 10.90 & & 664.57 & 138.00 & 1.15 & 0.36 & b & $\mathrm{P}$ \\
\hline 222582 & 113.30 & & 6171.45 & 1246.00 & 0.99 & 0.36 & b & $\mathrm{P}$ \\
\hline
\end{tabular}

Remarks: P: Stars with planets as in FV05; S: Stars without planets as in FV05; SB: Spectroscopic Binaries; RV: Stars with RV linear trends (see Nidever et al. 2002); $\Delta \mu$ : Stars with discrepant proper motion in Hipparcos and Tycho II (see Makarov \& Kaplan 2005); G: Stars with accelerating proper motions in Hipparcos. (see Makarov \& Kaplan 2005).

References: HD 3074: Allen et al. (2000); HD 4614: Worley \& Heintz (1983); HD 4747: Nidever et al. (2002); HD 7693: Nordström et al. (2004); HD 10360 - HD 10361: Worley \& Heintz (1983); HD 11964: Allen et al. (2000); HD 13445: Lagrange et al. (2006); Desidera \& Barbieri (2007); HD 13507: Perrier et al. (2003); HD 13531: Metchev (2006); HD 13612: Worley (1967a); Duquennoy \& Mayor (1991); HD 16141: Desidera \& Barbieri (2007); Mugrauer et al. (2004); HD 16160: Allen et al. (2000); Golimowski et al. (2000); HD 16895: Worley \& Heintz (1983); HD 18445: Duquennoy \& Mayor (1991); Halbwachs et al. (2000); Zucker \& Mazeh (2001); HD 20782: Desidera \& Barbieri (2007); HD 23439: Allen et al. (2000); HD 27442: Chauvin et al. (2006); Desidera \& Barbieri (2007); HD 29836: Griffin \& Gunn (1981); HD 30649: Nidever et al. (2002); Dommanget \& Nys (2002); HD 31412: Nidever et al. (2002); Gliese \& Jahreiß (1991); HD 35956: Vogt et al. (2002); HD 38529: Reffert \& Quirrenbach (2006); Desidera \& Barbieri (2007); HD 39587: Nidever et al. (2002); HD 43587: Vogt et al. (2002); Duquennoy \& Mayor (1991); Salim \& Gould (2003); HD 64468: Vogt et al. (2002); HD 65430: Nidever et al. (2002); HD 65907: Tokovinin (1997); HD 72760: Metchev (2006); HD 77407: Mugrauer et al. (2004), Metchev (2006); HD 86728: Gizis et al. (2000); Lépine \& Bongiorno (2007); HD 90839: Gliese \& Jahreiß (1991); Duquennoy \& Mayor (1991); HIP 52940: Nidever et al. (2002); HD 92222: Fabricius et al. (2002), this paper; HD 97334: Burgasser et al. (2005); HD 101177: Duquennoy \& Mayor (1991); Salim \& Gould (2003); HD 111398: Lépine \& Bongiorno (2007); HD 120066: Allen et al. (2000); Gould \& Chanamé (2004); HD 120136: Raghavan et al. (2006); Desidera \& Barbieri (2007); HD 120237: Allen et al. (2000); HD 120780: Makarov \& Kaplan (2005); HD 122742: Nidever et al. (2002); HD 128620 - HD 128627:Worley \& Heintz (1983); HD 131156: Worley \& Heintz (1983); Duquennoy \& Mayor (1991); HD 131511: Nidever et al. (2002); HD 134440/39:Allen et al. (2000); HD 135101: Desidera et al. (2004); HD 139323: Worley \& Heintz (1983); HD 139477: Lépine \& Bongiorno (2007); HD 140913: Nidever et al. (2002); HD 146362 B: Worley \& Heintz (1983); Tokovinin (1997); HD 150554: Metchev (2006); HD 156274: Worley \& Heintz (1983); HD 161797: Worley \& Heintz (1983); Nidever et al. (2002); Wittenmyer et al. (2006); Makarov \& Kaplan (2005); HD 167215: Makarov \& Kaplan (2005); HD 169822: Vogt et al. (2002); HD 174457: Nidever et al. (2002); HD 178911 B: Tokovinin et al. (2000); HD 184860: Vogt et al. (2002); HD 185395: Lépine \& Bongiorno (2007); HD 187691: Duquennoy \& Mayor (1991); HD 190360: Allen et al. (2000); Desidera \& Barbieri (2007); HD 190406: Liu et al. (2002); HD 191408: Allen et al. (2000); HD 195019: Allen et al. (2000); Desidera \& Barbieri (2007); HD 197076: Duquennoy \& Mayor (1991); HD 196885: Chauvin et al. (2006); HD 198387: Makarov \& Kaplan (2005); HD 206860: Luhman et al. (2006); HD 208776: Nidever et al. (2002); HD 213519: Lépine \& Bongiorno (2007); HD 219542: Desidera et al. (2004); HD 219834: Tokovinin (1997); HD 221830: Allen et al. (2000). 


\section{Appendix A: Comments on individual objects}

\section{A.1. List of included binaries}

- HD 3651: planet outside the UD limits. The companion is a cool brown dwarf.

- HD 7693: Hipparcos lists a companion at 0.9 arcsec ( $\sim 19$ AU). Furthermore, the star is listed in CCDM as the wide companion of HD 7788, with a separation of 319 arc$\sec (\sim 6731 \mathrm{AU})$. The values of parallaxes and proper motion in right ascension would suggest a physical bounding, and the RV values reported by Nordström et al. (2004) seems to confirm this hypothesis. But the RV values for HD 7788 has large errors, and the proper motion in declination reported by Hipparcos are in disagreement. Probably this peculiarity could be explained by considering that both HD 7693 and HD 7788 are close binaries themselves. In fact Hipparcos lists a companion at 0.9 arcsec for the first star, and one at 5 arcsec $=105 \mathrm{AU}$, for the last one. Because we are interested in the effect of the binarity on the planetary formation/evolution, we will take only the closest companion to HD 7693 into account.

- HD 11964: planet outside the UD limits.

- HD 13445 (GL 86): the companion was discovered by Els et al. (2001) and it was classified as a brown dwarf, but successive work shows that the secondary is a $\sim 0.5 M_{\odot}$ white dwarf (Mugrauer \& Neuhäuser 2005; Lagrange et al. 2006). Desidera \& Barbieri (2007) described a possible evolution of the system during the mass-loss phase of the originally more massive star. The white dwarf companion is responsible for the observed RV and astrometric trends.

- HD 13507: suspected for some time of harboring a planet, but the later measurements obtained with ELODIE invalidated this interpretation and instead revealed a classical spectroscopic binary velocity curve, caused by a low-mass ( $m \sin i \sim 50 M_{J}$ ) companion (Perrier et al. 2003). Adaptive optics imaging did not directly detect the companion.

- HD 13612: triple system. AB is a CPM pair. A is an SB2 (Duquennoy \& Mayor 1991) and the disagreement between spectral and photometric parallaxes of A and B are probably due to its nature. An additional component at 2:9 is optical (Worley 1967b); the component included in the UD sample is HD 13612 B and, because the mass of this star is not listed in VF05, we derive it according to the Reid \& Gizis (1997) and Delfosse et al. (2000) mass-luminosity calibration.

- HD 16141: planet outside the UD limits.

- HD 16160 = GL105: triple system composed of an inner pair and another wide companion $(\rho=164.8 \operatorname{arcsec}=1200 \mathrm{AU}$, mass $\left.0.38 M_{\odot}\right)$. The orbit of the close pair was derived by Golimowski et al. (2000) ( $a=15 \mathrm{AU} e=0.75)$. The mass of the close companion is about $0.09 M_{\odot}$.

- HD 18143: triple system. Component B at 6.5 arcsec $=149$ $\mathrm{AU}$, component $\mathrm{C}$ at $43 \operatorname{arcsec}=985 \mathrm{AU}$.

- HD 18445: is a member of a quintuple system (component C). Components AB (HD 18455) form a visual binary with $P=147 \mathrm{yr}$ and $a=1.55 \operatorname{arcsec}=40 \mathrm{AU}$ (Worley $\&$ Heintz 1983), $\mathrm{C}$ is at 27.2 arcsec $\sim 700 \mathrm{AU}$ and is a spectroscopic binary with minimum mass in the brown dwarf range ( $m \sin i=0.042 M_{\odot}$ according to Zucker \& Mazeh 2001). Halbwachs et al. (2000) demonstrated that the pair is close to face-on and derived a mass of $0.176 M_{\odot}$. The close companion was also visually resolved by Beuzit et al. (2004) at $0.1 \operatorname{arcsec}=2.6 \mathrm{AU}$. A further CPM companion (D) is at a projected separation 5.090 arcsec $=130 \mathrm{AU}$ from $\mathrm{C}$.
- HD 20782: listed as a binary in the CCDM catalog. The companion is HD 20781. Desidera \& Barbieri (2007) suggest a physical association and consider HD 20782 and HD 20781 as a very wide CPM pair.

- HD 23439: triple system. The secondary is a spectroscopic binary (period $=49$ days, masses $0.74+0.12 M_{\odot}$ ).

- HD 27442 ( $\epsilon$ Ret): a companion at $\sim 13$ arcsec $~ 237$ AU is included in WDS and was confirmed by Chauvin et al. (2006). HD 27442 B is probably a white dwarf with a mass of about $0.6 M_{\odot}$ (see Desidera \& Barbieri 2007).

- HD 29836: triple system. HD 285970 is a wide companion of the star included in the UD sample. HD 285970 is also a short-period spectroscopic binary (Griffin \& Gunn 1981). Another companion to HD 29836 at 100 arcsec is listed in CCDM, probably optical.

- HD 30649: CCDM lists a companion at 3.37 arcsec = $101 \mathrm{AU}$, but probably this is not the cause of the linear trend reported by Nidever et al. (2002).

- HD 31412: additional common proper motion companion (CNS3, Lépine \& Bongiorno 2007) at $22 \operatorname{arcsec}=792 \mathrm{AU}$; it should not be responsible for the observed RV trend.

- HD 35956: triple system. The primary (the star in the UD sample) is a spectroscopic and astrometric binary (Vogt et al. 2002). An additional companion $\left(M=0.44 M_{\odot}\right)$ is at a projected separation of $99 \operatorname{arcsec}=2860 \mathrm{AU}$.

- HD 38529: it hosts two low-mass companions with projected masses of $0.78 M_{J}$ for the inner companion and $12.70 M_{J}$ for the outer companion. This star shows an astrometric motion (it is marked with G-Flag in the Hipparcos Catalogue) which is probably due to the presence of the outer companion (HD 38529 C) for which Reffert \& Quirrenbach (2006) derived a mass of $37_{-19}^{+36} M_{J}$, clearly into the brown dwarf regime. A stellar companion at very wide separation is also present. The classification of this star as a two-planet host with a wide stellar companion or a single-planet host with a brown dwarf and another wide companion is ambiguous (Desidera \& Barbieri 2007).

- HD 43587: triple system. The primary (in the UD sample) is a spectroscopic binary (Vogt et al. 2002). An additional component $\left(M \sim 0.30 M_{\odot}, \rho=95 \operatorname{arcsec}=2860 \mathrm{AU}\right.$ was shown to be physically associated (Duquennoy \& Mayor 1991). And other 3 faint companions are listed, probably all optical (NLTT);

- HD 53705- HD 53706: another distant companion (K5, mass $0.69)$ at $185 \operatorname{arcsec}=3000$ AU.

- HD 65907: triple system. The secondary, at a projected separation of 60 arcsec from the primary, is itself a close visual binary (projected separation $37 \mathrm{AU}$ ). Individual masses 0.63 and $0.35 M_{\odot}$.

- HD 72760: Metchev (2006) reports a companion of $0.13 M_{\odot}$ at a separation of $0.96 \operatorname{arcsec}=21 \mathrm{AU}$, which is probably responsible of the astrometric trend found by Makarov \& Kaplan (2005).

- HD 77407: 0.30 $M_{\odot}$ companion at 1.6 arcsec = 50 AU separation imaged by Calar Alto Adaptive optic system ALFA and confirmed as physically bound to the primary by a multiepoch, high-resolution spectrum (Mugrauer et al. 2004) that shows a long-term radial velocity trend for HD $77407 \mathrm{~A}$. The companion was also confirmed by Metchev (2006).

- HD 86728: the M dwarf companion is overluminous with an high activity level. Gizis et al. (2000) suggest it is itself a close binary. 
- HD 90839: CPM pair. There is a third companion, HD 89862, but it is not physical (see Gliese \& Jahreiß 1991; Duquennoy \& Mayor 1991).

- HD 92222 A: it is not included in the Hipparcos Catalogue. For this reason the mass of this star is not listed in VF05. We derived a photometric distance assuming that both components are on the main sequence; and by using the isochrones by Girardi et al. (2002) we found $d=90 \mathrm{pc}$ and $M_{A}=1.09$ $M_{\odot}, M_{B}=1.05 M_{\odot}$.

- HIP 52940 - HIP 52942: triple system. The primary is a spectroscopic binary discovered by Nidever et al. (2002). Both components are in the UD sample and both masses are derived according with Reid \& Gizis (1997) and Delfosse et al. (2000) mass-luminosity calibrations, because VF05 do not list any mass value for these objects.

- HD 97334: a close pair of brown dwarfs (separation 1.5 AU, total mass $=0.05 M_{\odot}$ ) is at a projected separation of about 2000 AU from the primary (Burgasser et al. 2005).

- HD 99492 B: planet outside the UD limits.

- HD 101177: triple system. The secondary is a spectroscopic binary $\left(P=23 \mathrm{~d}\right.$, masses $=0.74$ and $\left.0.31 M_{\odot}\right)$. Component $\mathrm{C}$ is optical as the other two companions listed most likely are (NLTT).

- HD 114729: planet outside the UD limits.

- HD $120136=\tau$ Boo: the orbital solution by Hale (1994) is very preliminary. Another L dwarf companion candidate at 42 arcsec $=664 \mathrm{AU}$ has been reported by Pinfield et al. (2006). The physical association has yet to be confirmed.

- HD 120780: CCDM lists a companion at $6 \operatorname{arcsec}=98 \mathrm{AU}$ $\left(M=0.53 M_{\odot}\right)$, but probably this is not responsible for the astrometric signature reported by Makarov \& Kaplan (2005).

- HD 128620-HD128621 ( $\alpha$ Cen): Triple system: another low mass companion (Proxima Cen) at very wide separation (10000 AU).

- HD $131156=\xi$ Boo: visual binary $(\rho=4.9$ arcsec $=33$ AU, $P=152$ yr) (see Worley \& Heintz 1983). The visual companion explains the long-term radial velocity trend with a significant curvature detected by Wittenmyer et al. (2006). There are two additional components listed in WDS: C $(V=12.6, \rho=66.7 \operatorname{arcsec}=447$ AU, from CCDM $)$, which is optical (Duquennoy \& Mayor 1991), and D $(V=9.6$, $\rho=49 \operatorname{arcsec}=328.3 \mathrm{AU})$.

- HD 131977: quadruple system. The secondary (HD 131976) is itself a binary with $a=0.9$ AU and individual masses 0.57 and $0.38 M_{\odot}$. The fourth component is the brown dwarf GL $570 \mathrm{~B}$ at a projected separation of $1500 \mathrm{AU}$ (Burgasser et al. 2000).

- HD 137778: wide visual pair with a similar component. Only the secondary (HD 137778) is included in the UD sample. The primary (HD 137763) is a spectroscopic binary with extreme eccentricity ( $e=0.975$; Duquennoy et al. 1992).

- HD 139323: hierarchical triple system. The companion HD 139341 is a visual binary with $a=18 \mathrm{AU}$ and individual masses of 0.74 and $0.81 M_{\odot}$.

- HD 145958: another companion candidate has been reported at 0.2 arcsec $=5$ AU by Tokovinin (1997) but it needs confirmation (no RV variations observed). Preliminary binary orbit of the wide pair in WDS (grade 4).

- HD 146362: member of a hierarchical multiple system. The star included in the UD sample is orbiting the nearly equalmass double-lined spectroscopic binary HD 146361 (period 1.1 days, individual masses 1.10 and $1.09 M_{\odot}$ ). Preliminary orbit of the wide pair in WDS. Another CMP companion, a faint M3V dwarf, is at $633 \operatorname{arcsec}=14000 \mathrm{AU}$.
- HD 161797 ( $\mu$ Herculis): astrometric orbit by Heintz (1994) (period 65 years). The companion was also identified using adaptive-optics imaging by Turner et al. (2001) and Debes et al. (2002). Radial velocity monitoring revealed a long term trend with significant curvature (Nidever et al. 2002; Wittenmyer et al. 2006). The star is also listed as a $\Delta \mu$ binary in Makarov \& Kaplan (2005). Another companion, $\mu^{2}$ Her B, lies at $34 \operatorname{arcsec}=285$ AU and is itself a visual binary with period $43.2 \mathrm{yr}$ (WDS).

- HD 167215: CCDM lists a companion with $\rho=54$ ". 8 ( $M=$ $\left.1.05 M_{\odot}\right)$, but is not expected to be responsible of the astrometric signature reported by Makarov \& Kaplan (2005).

- HD 169822: triple system. The star included in the UD sample is a spectroscopic binary detected during the Keck survey. Vogt et al. (2002) derived a combined spectroscopic and astrometric solution ( $\left.M=0.30 M_{\odot}, a=0.84 \mathrm{AU}\right)$. HD 169889 , at $608.4 \operatorname{arcsec}=16426.8 \mathrm{AU}$, is a CPM star. The revised distance of HD 169822 by Vogt et al. (2002) (32 pc), coupled with the common RVs for the two stars (Nordström et al. 2004) makes the indication of a physical association stronger.

- HD 178911: triple system (Tokovinin et al. 2000).

- HD 184860: triple system. The star included in the UD sample is a spectroscopic binary (Vogt et al. 2002). The projected mass of the companion is in the brown dwarf range $\left(32 M_{J}\right)$. An additional companion is at 5.0 arcsec $=151 \mathrm{AU}$.

- HD 187691: three components listed in CCDM/WDS. AC is a CPM pair, B is optical, $\mathrm{C}$ is physical (Duquennoy \& Mayor 1991).

- HD 190067: Turner et al. (2001) listed a companion at 2.86 arcsec $=55.19 \mathrm{AU}$, confirmed by Chakraborty et al. (2002) that reported $m \sim 0.08-0.10 M_{\odot}$.

- HD 190360: Planet outside the UD limits.

- HD 190406 (15 Sge): A brown dwarf companion $(\rho=$ $0.79 \operatorname{arcsec}=14 \mathrm{AU}$ ) was found with high-resolution imaging made using adaptive optics at the Gemini-North and Keck telescopes (Liu et al. 2002). The primary shows a longterm radial velocity trend that confirms that HD $190406 \mathrm{~B}$ is physical, with a minimum mass of $M=48 M_{J}$.

- HD 196885: Chauvin et al. (2006) identified at about 0.7 arcsec a relatively bright companion candidate: HD $196885 \mathrm{~B}$, that is likely to be a late K-dwarf, with a mass of $0.6 M_{\odot}$, orbiting HD $196885 \mathrm{~A}$ at a projected physical distance of 25 AU. HD 196885 was also classified as a $\Delta \mu$ binary by Makarov \& Kaplan (2005). The astrometric signature is probably due to HD 196885 B. A planetary companion was also claimed from the Lick RV Survey ${ }^{8}$, but it is not confirmed in the recent work by Butler et al. (2006). The companion $\mathrm{BD}+104351 \mathrm{~B}$, listed in WDS and CCDM, is probably optical.

- HD 197076: the companion listed as B in WDS and CCDM $\left(m_{v}=11.6\right.$ and $\left.\rho=94 \operatorname{arcsec}=1974 \mathrm{AU}\right)$ is optical, but the really bound companion is component $\mathrm{C}(\rho=125 \operatorname{arcsec}=$ 2625 AU) (Duquennoy \& Mayor 1991).

- HD 198387: CCDM lists a companion with $\rho=12.2=$ 511 AU, but probably this is not responsible for the astrometric signature reported by Makarov \& Kaplan (2005).

- HD 215578: the mass of this star is not listed in VF05, so we derive the value listed in Table 8 according with Reid \& Gizis (1997) and Delfosse et al. (2000) mass-luminosity calibration.

\footnotetext{
${ }^{8}$ http//exoplanets.org/esp/hd196885/hd196885.shtml
} 
- HD 219542: the masses of the components of this star were taken from Desidera et al. (2004), as there is some confusion in the identification of the components in the photometry used by VF05 to derive stellar masses (for one of the components, the joint $\mathrm{A}+\mathrm{B}$ magnitude is used).

- HD 219834: hierarchical triple system. The primary is a spectroscopic binary (period $=6.2 \mathrm{yr}$, individual masses of 0.90 and $\left.0.04 M_{\odot}\right)$. The secondary is the star included in the UD sample and the mass was apparently derived in VF05 from the magnitude of the much brighter primary. We assumed the mass given in the on-line version of the MSC (Tokovinin 1997).

\section{A.2. Unconfirmed binaries}

- HD 23249 ( $\delta$ Eri): belongs to the group of nearest stars and is classified as a weakly active and X-ray soft source (Huensch et al. 1998). Fisher et al. (1983) tried to detect a periodic variation in the photometric data and suggest that $\delta$ Eri could be classified as an RS CVn star: that is, a F-G binary star having a period $<14$ days, with chromospheric activity and with a period of rotation synchronized with its orbital period (Linsky 1984), giving the star high rotational velocity inducing strong activity. This contrasts with the low level of activity detected by Thévenin et al. (2005) and the lack of radial velocity variations, making this classification doubtful.

- HD 52265, HD 154857, HD 179949: first epoch observations by Chauvin et al. (2006) revealed companion candidates, but the physical association with the planet hosts has not yet been confirmed;

- HD 102158: the presence of a common proper motion companion at 1175.2 arcsec was proposed by Lépine \& Bongiorno (2007). The corresponding projected separation (61000 AU) is much larger than any other companion in Table 8, so it is not considered here.

- HD 107213: Lépine \& Bongiorno (2007) propose that this star form a wide (projected separation 546 arcsec) common proper motion pair with BD+28 $2103=$ HIP 60061 . However, the discrepant RVs for the two stars (Latham et al. 2002; Nordström et al. 2004) argue against a physical association.

- HD 117176: an L dwarf companion candidate at 848 AU has been reported by Pinfield et al. (2006). The physical association has yet to be confirmed.

- HD 168443: as for HD 38529, there is an ambiguity about the physical classification of the companion as a massive planet or brown dwarf (see Desidera \& Barbieri 2007).

- HD 217107: the presence of the companion listed in WDS is not confirmed by recent adaptive optics searches. See Desidera \& Barbieri (2007) for discussion and references. 